\title{
Comparative Efficacy of Linaclotide Versus Other Oral Constipation Treatments in Chronic Constipation: a Network Meta-analysis
}

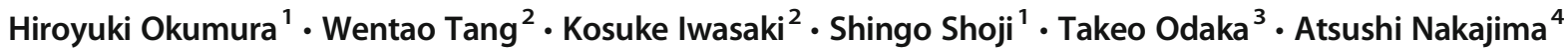

Accepted: 18 August 2020 / Published online: 29 August 2020

(C) The Author(s) 2020

\begin{abstract}
This systematic literature review and network meta-analysis (NMA) indirectly compared the Japanese standard dose of linaclotide $500 \mu \mathrm{g}$ with other oral chronic constipation (CC) treatments. PubMed, Cochrane-CENTRAL, IchushiWeb, and ClinicalTrials.gov were systematically searched for eligible randomized controlled trials of 43 oral drugs approved globally for $\mathrm{CC}$, including irritable bowel syndrome with constipation (IBS-C) and opioid-induced constipation (OIC). The mean difference (95\% credible interval) in change from baseline in weekly number of spontaneous bowel movements (SBM) was compared between linaclotide $500 \mu \mathrm{g}$ (unapproved in OIC) and other treatments using Bayesian methodology. Fifty-two publications (54 trials) involving 47 treatments (16 drugs, different doses of the same drug treated as different treatments) were included in the NMA. Despite including various drugs/doses, for the mean difference in weekly SBM change, linaclotide $500 \mu \mathrm{g}$ was statistically significantly more efficacious than other drugs/doses (vs $500 \mu \mathrm{g}$ linaclotide) including the following: placebo $(-1.907 ;-2.568,-1.237)$; lubiprostone $16 \mu \mathrm{g}(-2.090 ;-3.226,-0.968)$; methylnaltrexone $150 \mathrm{mg}(-1.807 ;-3.126,-0.491), 300 \mathrm{mg}(-1.411 ;-2.722$, $-0.096)$, and $450 \mathrm{mg}(-1.405 ;-2.708,-0.097)$; naloxegol $5 \mathrm{mg}(-2.074 ;-4.001,-0.131)$ and $12.5 \mathrm{mg}(-1.329$; $-2.347,-0.318)$; and tegaserod $4 \mathrm{mg}(-1.133 ;-2.059,-0.207)$ and $12 \mathrm{mg}(-1.024 ;-1.822,-0.228)$, and statistically significantly less effective than linaclotide $600 \mu \mathrm{g}$ non-approved dose $(1.159 ; 0.123,2.199)$ and bisacodyl $10 \mathrm{mg}(2.979 ; 1.723,4.233)$. These findings provide relative efficacy data for linaclotide $500 \mu \mathrm{g}$ vs other constipation drugs/doses regarding improving weekly SBM in CC and IBS-C and may inform clinical decisionmaking for constipation treatments.
\end{abstract}

Keywords Constipation $\cdot$ Linaclotide $\cdot$ Network meta-analysis $\cdot$ Systematic literature review

This article is part of the Topical Collection on Medicine

Hiroyuki Okumura was an employee of Astellas Pharma Inc. at the time of the study

Wentao Tang was an employee of Milliman at the time of the study

Electronic supplementary material The online version of this article (https://doi.org/10.1007/s42399-020-00467-x) contains supplementary material, which is available to authorized users.

Hiroyuki Okumura

hm-okmra@zc4.so-net.ne.jp

Medical Affairs, Astellas Pharma, Inc., Tokyo, Japan

Milliman, Tokyo, Japan

Odaka Medical and Gastrointestinal Clinic, Chiba, Japan

4 Department of Gastroenterology and Hepatology, Yokohama City University Graduate School of Medicine, Yokohama, Japan

\section{Introduction}

Chronic constipation (CC), including functional constipation and irritable bowel syndrome with constipation (IBS-C), affects approximately $14 \%$ of adults globally [1], negatively impacts the quality of life of patients, and increases healthcare costs [2-5]. Chronic constipation is characterized by infrequent bowel movements, hard stools, feeling of incomplete evacuation, abdominal discomfort or pain, and bloating sensation [6]. The initial treatment strategy for constipation usually includes non-pharmacological approaches such as dietary fiber, changes in life habits, or exercise, followed by pharmacological agents in non-responsive individuals [7, 8]. Several classes of pharmaceutical agents are available for treatment of different types of constipation such as bulking agents (e.g., ispaghula, wheat), osmotic laxatives (polyethylene glycol [PEG], lactulose), stimulant laxatives (e.g., bisacodyl), stool 
softeners and lubricants, prokinetic agents (e.g., prucalopride), and secretagogues (e.g., lubiprostone) [7, 8]. Despite the abundance of treatment options available for the different constipation types, nearly $50 \%$ of patients are dissatisfied with current treatments because of lack of efficacy and unwanted side effects [9].

In Japan, the prevalence of CC, including IBS-C, is higher than global estimates (approximately 28\%), but there is little epidemiological or humanistic information on $\mathrm{CC}$ currently available [10]. Currently, magnesium oxide, followed by stimulant laxatives, are widely used for treatment of $\mathrm{CC}$ in Japan [11]. Recently, linaclotide, a first-in-class, minimally absorbed oligo peptide with guanylate cyclase-C agonistic activity [12], was approved for IBS-C followed by $\mathrm{CC}$ in Japan [13]. Based on the results of dose-determining clinical studies conducted in Japan and the United States (US), the approved standard dose in Japan is higher $(500 \mu \mathrm{g})$ than the doses approved for CC (72 $\mu \mathrm{g}$ and $145 \mu \mathrm{g})$ and IBS-C $(290 \mu \mathrm{g})$ in the US $[13,14]$. However, the relative efficacy of $500 \mu \mathrm{g}$ linaclotide in comparison to the available treatment modalities for CC in Japan and globally is unknown because of a lack of head-to-head comparison trials.

In clinical practice, selection of the most appropriate therapy for CC is challenging due to the lack of direct comparisons between the available constipation drugs. Most published trials on constipation treatments are placebo-controlled studies, limiting the ability to compare active treatments $[15,16]$. A valid statistical estimate of the comparative efficacy of different treatment modalities can be achieved using a network meta-analysis (NMA) that combines direct head-to-head evidence and indirect comparative evidence [17-20]. An NMA of different treatments for $\mathrm{CC}$ has recently been published [21]; however, it did not include patients with IBS-C, was limited to evidence primarily from Western countries, and included a limited number of constipation treatments. The objective of this study was to perform a systematic literature review (SLR) and NMA to compare the efficacy of linaclotide $500 \mu \mathrm{g}$ to other available treatment modalities (including other linaclotide doses) for CC, including IBS-C.

\section{Methods}

\section{Study Design}

An SLR and NMA of global (including Japanese) clinical trials on $\mathrm{CC}$ was conducted to compare $500 \mu \mathrm{g}$ linaclotide with other constipation treatments. The conduct of the study was based on a protocol that has been published (Registration Number: CRD42018111737) in the PROSPERO International prospective register of systematic reviews [22]. Identification of studies on CC treatments, the literature search strategy, and the analysis of risk of bias of included studies were performed using the
Cochrane Handbook for Systematic Reviews of Intervention [23]. The results have been reported according to the guidelines on Preferred Reporting Items for Systematic Reviews and MetaAnalyses (PRISMA) extension statement for reporting systematic reviews incorporating an NMA [24].

\section{Search Strategy}

The SLR was conducted using the databases PubMed, Ichushi-Web (a Japanese bibliographic database maintained by Japan Medical Abstracts Society), Cochrane-CENTRAL, and ClinicalTrials.gov up to August 8, 2017. The majority of the Cochrane-CENTRAL records were taken from MEDLINE and EMBASE, but records were also derived from other published and unpublished sources [25].

To establish a broad network among constipation treatments, approximately 43 oral drug treatments for constipation were considered for inclusion in the analysis. The comparator drugs, which were identified from the World Health Organization Anatomical Therapeutic Chemical classification system, World Gastroenterology Organisation Global Guidelines, and Japanese guidelines for the treatment of constipation, included the following: linaclotide, liquid paraffin, docusate sodium (sodium dioctyl sulfosuccinate), oxyphenisatine, bisacodyl, dantron, phenolphthalein, castor oil, senna glycosides (sennosides), cascara (casanthranol), sodium picosulfate, bisoxatin, ispaghula (psylla seeds), ethulose, sterculia, linseed, methylcellulose, Triticum (wheat fiber), polycarbophil calcium, magnesium carbonate, magnesium oxide, magnesium peroxide, magnesium sulfate, magnesium hydroxide, lactulose, lactitol, sodium sulfate, pentaerithrityl, macrogol (or PEG), mannitol, sorbitol, sodium phosphate, magnesium citrate, sodium tartrate, methylnaltrexone bromide, alvimopan, naloxegol, naloxone, lubiprostone, prucalopride, tegaserod, plecanatide, and mosapride. The common search terms used were constipation, IBS-C, opioid-induced constipation (OIC), along with the generic and brand names of the above 43 selected treatments, and were searched in all fields. The term OIC was included because some pre-determined drug therapies were approved for OIC in addition to CC and IBS-C (e.g., lubiprostone). Therefore, in addition to treatments for $\mathrm{CC}$ and IBS-C, the network was expanded to include studies that also had an OIC treatment arm. Multiple different combinations of these treatments using "and/or" were used. No limits were applied for language, publication date, or publication status; foreign-language publications were translated. The detailed search strategy used for PubMed is shown in Table S1. The search strategies for all databases were similar and were adapted for each database.

\section{Study Selection and Data Extraction}

The eligibility criteria involved limiting all searches to randomized controlled trials (RCTs) and quasi-randomized trials 
with data for the primary outcome and including all trials conducted in Japan and other countries that included patients $>18$ years with CC including IBS-C and OIC. The following studies were excluded: observational studies and studies other than clinical trials or without a control group, studies on patients with organic constipation, any studies assessing constipation treatments other than the 43 selected oral drugs, treatments administered rectally, studies on diagnosis and prevention of constipation, and any studies not reporting the primary outcome measure or with incomplete outcomes.

All studies retrieved from the literature search were assessed for inclusion by two independent reviewers (WT and KI). Reference lists of retrieved studies were also manually searched to identify studies not retrieved by the electronic literature search. After removal of duplicates, studies were screened for eligibility first using titles and abstracts and second using the full text. Any disagreements were resolved by consensus, and resolution of disagreements was finally confirmed by HO. Extraction of data from the eligible trials was conducted by WT and KI. Only published data were used for this analysis. Missing data for any study endpoint were not included in the analysis. Besides the study endpoint, the time point of endpoint data reported, patient characteristics, and the constipation type reported in the eligible studies were also extracted.

\section{Assessment of Risk of Bias}

Risk of bias of each trial was conducted in accordance with the Cochrane Handbook for Assessing the Risk of Bias [26]. The risk of bias was categorized as high, low, or unclear.

\section{Outcomes Assessed}

The primary endpoints for this study were the change from baseline in weekly number of spontaneous bowel movements (SBM), complete spontaneous bowel movements (CSBM), change in severity scores for abdominal bloating and abdominal discomforts, and change in scores for stool characteristics and patient quality of life. The secondary endpoint was treatment-related adverse events. However, only SBM is reported here because of insufficient or poor-quality data for the other outcomes in the selected studies.

\section{Network Meta-analysis}

Outcome data extracted from each of the eligible clinical trials were used to conduct the NMA to indirectly compare the different constipation interventions, including placebo, with linaclotide $500 \mu \mathrm{g}$. The treatment modalities included in the NMA were placebo, linaclotide, lubiprostone, plecanatide, PEG, prucalopride, lactulose, bisacodyl, ispaghula, wheat, lactitol, methylnaltrexone, alvimopan, naloxegol, naloxone, and tegaserod. Trials on combination therapies were not included.
Trials studying different doses of a single treatment (e.g., 16, 32 , and $48 \mu \mathrm{g}$ lubiprostone; 1,2 , and $4 \mathrm{mg}$ prucalopride; and 0.5 and $1 \mathrm{mg}$ alvimopan) or non-approved treatment dosages (e.g., linaclotide $1000 \mu \mathrm{g}$ ) were included in the treatment network. In general, different doses of a single treatment were considered as separate treatment modalities in the network. However, to simplify the network, small differences in drug dosages considered to be clinically equivalent (e.g., 10 and $10.35 \mathrm{~g}$ PEG; 579 and $600 \mu \mathrm{g}$ linaclotide) were pooled into the same drug group [27, 28]. For linaclotide, the following doses were assessed, and those considered clinically equivalent were pooled (with assistance from Ironwood Pharmaceuticals): 72 and $75 \mu \mathrm{g}, 145$ and $150 \mu \mathrm{g}, 290$ and $300 \mu \mathrm{g}$, and 579 and $600 \mu \mathrm{g}$. Similarly, for PEG, the 10-g and 10.35-g doses were considered clinically equivalent and were pooled.

An NMA based on the methodology proposed by White et al. [29] using Bayesian modeling was used to analyze the efficacy of all treatments in the network simultaneously. An arm-based approach (as proposed in the methodology by White et al.) was used, whereby for each trial, a model with a baseline treatment outcome, with other treatment outcomes as comparisons to the baseline treatment, was assessed. Non-informative prior distributions were used for the analyses using the Bayesian model. The main outcome parameter of the NMA was the mean difference and $95 \%$ credible interval (CrI) for the change in weekly number of SBM before and after linaclotide $500 \mu \mathrm{g}$ compared with each constipation treatment. Linaclotide $500 \mu \mathrm{g}$ was considered statistically significantly better than other treatments when the $95 \%$ CrI of the treatments was less than 0 and was considered statistically significantly worse than other treatments when the $95 \%$ $\mathrm{CrI}$ of the treatments was greater than 0 .

First, the network of different interventions including placebo was plotted in the NMA. Then, the NMA was conducted by fitting an inconsistency model. Consistency was defined as when the contrast effect of the same set of comparators did not change among different paths in the network. If the contrast effect changed, then inconsistency was considered to exist. Parameters of inconsistency were included in the inconsistency model and the null hypothesis of consistency was checked by globally testing all the inconsistency parameters using the global Wald test. If the consistency was not rejected by the global Wald test, then the NMA was conducted by fitting the consistency model without inconsistency parameters. Both inconsistency and consistency models were fitted by hierarchical Bayesian methodology. All NMA analyses were conducted using WinBUGS (version 1.4.3, MRC Biostatistics Unit, University of Cambridge, UK). The results of the NMA were assessed by two reviewers (HO and SS).

\section{Sensitivity Analyses}

The following sensitivity analyses were conducted: NMA limited to trials without high risk of bias, limited to $\mathrm{CC}$ (i.e., 
excluding trials of IBS-C and OIC), limited to CC and IBS-C (i.e., excluding trials of $\mathrm{OIC}$ ), and trials with average baseline weekly SBM less than 3 (severe constipation, i.e., excluding trials with mild-to-moderate constipation).

\section{Results}

\section{Study Inclusion}

Of the 1577 publications/trial articles retrieved and screened for inclusion, 52 publications (54 trials) were eligible and included in the NMA (Fig. 1). Manual searching identified 4 trials (phase 2 and 3 results of linaclotide in Japan, ClinicalTrials.gov identifiers: NCT01714843, NCT02316899, NCT02425722, and NCT02809105) that were accepted for publication at the time of this study and have since been published [30-33]. After removal of duplicates, trials were excluded if they were not aimed at studying the treatment effects of $\mathrm{CC}$, had interventions not included in the pre-determined 43 oral drug list, did not report change in SBM before and after treatment as the endpoint, included patients below the age of 18 years, were evaluating organic constipation, or were non-randomized trials or pre-clinical studies.

\section{Trial and Patient Characteristics}

An overview of the study characteristics of the 52 publications (54 trials) for which outcome data were collected is shown in
Table 1. Most included studies were placebo-controlled studies; only 3 studies compared active treatments [38, 60, 68] and 2 others compared different doses of the same active treatments $[40,65]$. Of the 52 publications, 23 compared different dosages of the same active treatment with placebo as a control group $[32-34,40,43,47,49,51,53,55,57-59,61,62,64,65,67,72$, $73,75,76,78]$. The sample size in the trials ranged from 20 patients to 1519 patients. A total of 22,733 patients with constipation (including CC, IBS-C, and OIC) were included across all trials. Although traditional treatments such as magnesium oxide were initially selected, they were not included for analysis in the NMA either due to limited and low-quality evidence or not meeting the inclusion criteria for age.

Overall, there was a low risk of bias within each of the included trials (Fig. S1) and across all trials (Fig. 2). Of the 54 trials, the risk of bias for one trial could not be assessed because the full text could not be obtained [71]. Randomization sequences were adequate and clearly reported in the remaining 53 trials (100\%), and blinding of participants and personnel were reported in approximately $94.3 \%$ of trials. A high risk of bias was evident for a relatively small proportion of the included trials, primarily because of a lack of blinding of outcome assessments (3 trials, 5.7\%). There was an unclear risk of bias for allocation concealment (39 trials, $73.6 \%$ ) and selective reporting (31 trials, $58.5 \%$ ).

The patient characteristics across the included studies are shown in Table S2. The average patient age in the studies ranged from 33.7 to 76.4 years, and the baseline SBM ranged from 0.9 to 7.1. Patient age was not reported in 2 studies [45,

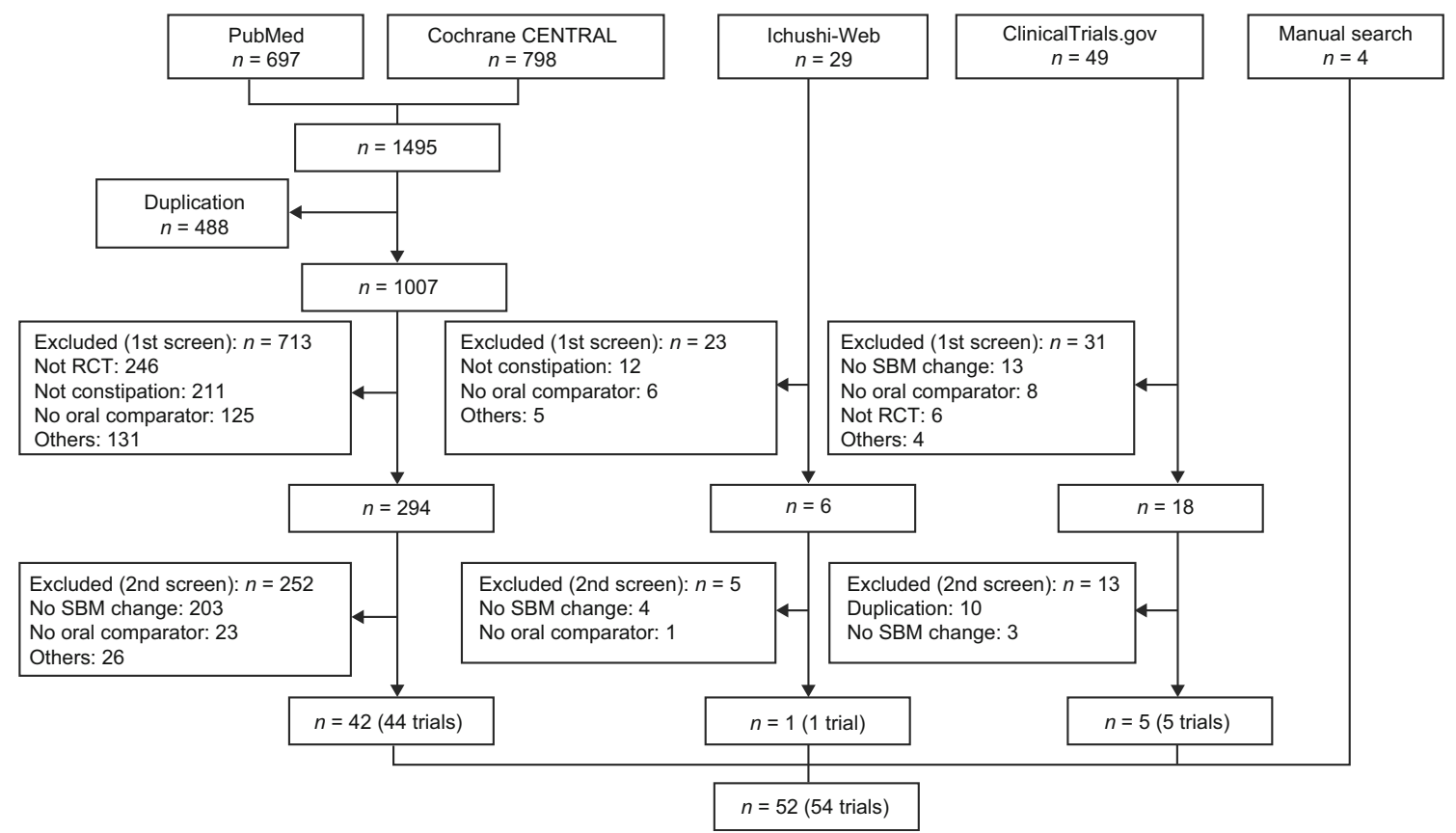

Fig. 1 Flow chart for study selection. Exclusion criteria for the first level of screening were no outcome data for effectiveness, safety, satisfaction, or quality of life of patients. The exclusion criterion for the second level of screening was no data for the change in SBM number before and after treatment. SBM, spontaneous bowel movement 
Table 1 Study characteristics of the included 52 studies (54 trials)

\begin{tabular}{|c|c|c|c|c|c|c|c|c|}
\hline Reference & Disease & Arm & Drug & $\begin{array}{l}\text { Dose/ } \\
\text { day }\end{array}$ & Patient number & $\begin{array}{l}\text { Change from } \\
\text { baseline in } \mathrm{SBM}^{\mathrm{a}}\end{array}$ & $\begin{array}{l}\text { Time point of } \\
\text { extracted data }\end{array}$ & $\begin{array}{l}\text { Baseline weekly } \\
\operatorname{SBM}^{\mathrm{b}}(\geq 3: 1 ;<3: 0 \\
\text { unknown: } \mathrm{N})\end{array}$ \\
\hline \multirow{3}{*}{$\begin{array}{l}\text { Andresen V, et al. } \\
\text { [34] }\end{array}$} & \multirow[t]{3}{*}{ IBS-C } & Arm 1 & Linaclotide & $100 \mu \mathrm{g}$ & 12 & $0.52 /$ day & 5 days & \multirow[t]{3}{*}{1} \\
\hline & & Arm 2 & Linaclotide & $1000 \mu \mathrm{g}$ & 12 & $0.9 /$ day & 5 days & \\
\hline & & Arm 3 & Placebo & NA & 12 & $0.22 /$ day & 5 days & \\
\hline \multirow{2}{*}{$\begin{array}{l}\text { Awad RA, et al. } \\
\text { [35] }\end{array}$} & \multirow[t]{2}{*}{ IBS-C } & Arm 1 & PEG & $10.35 \mathrm{~g}$ & 20 & 2.5/week & 30 days & \multirow[t]{2}{*}{0} \\
\hline & & Arm 2 & Placebo & & 22 & 2.8/week & 30 days & \\
\hline \multirow{2}{*}{$\begin{array}{l}\text { Badiali D, et al. } \\
\text { [36] }\end{array}$} & \multirow[t]{2}{*}{$\mathrm{CC}$} & Arm 1 & Triticum & $20 \mathrm{~g}$ & 12 & 3.8/week & 4 weeks & \multirow[t]{2}{*}{0} \\
\hline & & Arm 2 & Placebo & NA & 12 & $2.5 /$ week & 4 weeks & \\
\hline \multirow{2}{*}{$\begin{array}{l}\text { Barish CF, et al. } \\
\text { [37] }\end{array}$} & \multirow[t]{2}{*}{$\mathrm{CC}$} & Arm 1 & Lubiprostone & $48 \mu \mathrm{g}$ & 119 & 4.61/week & 1 week & \multirow[t]{2}{*}{0} \\
\hline & & Arm 2 & Placebo & $\mathrm{NA}$ & 118 & 2.49/week & 1 week & \\
\hline \multirow{2}{*}{$\begin{array}{l}\text { Bouhnik Y, et al. } \\
\text { [38] }\end{array}$} & \multirow[t]{2}{*}{$\mathrm{CC}$} & Arm 1 & PEG & $20 \mathrm{~g}$ & 32 & $0.28 /$ day & 4 weeks & \multirow[t]{2}{*}{1} \\
\hline & & Arm 2 & Lactulose & $20 \mathrm{~g}$ & 33 & $0.06 /$ day & 4 weeks & \\
\hline \multirow{2}{*}{$\begin{array}{l}\text { Chapman RW, } \\
\text { et al. [39] }\end{array}$} & \multirow[t]{2}{*}{ IBS-C } & Arm 1 & PEG & $26 \mathrm{~g}$ & 67 & $3.12 /$ week & 4 weeks & \multirow[t]{2}{*}{0} \\
\hline & & Arm 2 & Placebo & NA & 70 & $1.74 /$ week & 4 weeks & \\
\hline \multirow{4}{*}{$\begin{array}{c}\text { Chaussade S, } \\
\text { et al. [40] }\end{array}$} & \multirow[t]{4}{*}{$\mathrm{CC}$} & Arm 1 & PEG & $5.9 \mathrm{~g}$ & 67 & 5/week & 4 weeks & 0 \\
\hline & & Arm 2 & PEG & $10 \mathrm{~g}$ & 66 & 4.4/week & 4 weeks & \\
\hline & & Arm 3 & PEG & $11.8 \mathrm{~g}$ & 69 & 5.8/week & 4 weeks & \\
\hline & & Arm 4 & PEG & $20 \mathrm{~g}$ & 67 & 4.8/week & 4 weeks & \\
\hline Chey WD, et al. & IBS-C & Arm 1 & Linaclotide & $290 \mu \mathrm{g}$ & 401 & 4/week & 12 weeks & 0 \\
\hline$[41]$ & & Arm 2 & Placebo & NA & 403 & $1.3 /$ week & 12 weeks & \\
\hline Chey WD, et al. & IBS-C & Arm 1 & Tegaserod & $12 \mathrm{mg}$ & 172 & 2.31/week & 4 weeks & 1 \\
\hline$[42]$ & & Arm 2 & Placebo & NA & 164 & $1.49 /$ week & 4 weeks & \\
\hline Chey WD, et al. & $\mathrm{OIC}$ & Arm 1 & Naloxegol & $12.5 \mathrm{mg}$ & 211 & 2.56/week & 12 weeks & 0 \\
\hline [43] & & Arm 2 & Naloxegol & $25 \mathrm{mg}$ & 212 & $3.02 /$ week & 12 weeks & \\
\hline & & Arm 3 & Placebo & NA & 211 & $2.02 /$ week & 12 weeks & \\
\hline Chey WD, et al. & OIC & Arm 1 & Naloxegol & $12.5 \mathrm{mg}$ & 228 & 2.62/week & 12 weeks & 0 \\
\hline [43] & & Arm 2 & Naloxegol & $25 \mathrm{mg}$ & 226 & $3.14 /$ week & 12 weeks & \\
\hline & & Arm 3 & Placebo & NA & 231 & 2.1/week & 12 weeks & \\
\hline Christie J, et al. & $\mathrm{CC}$ & Arm 1 & Lubiprostone & $48 \mu \mathrm{g}$ & 37 & $3.59 /$ week & 4 weeks & 0 \\
\hline$[44]$ & & Arm 2 & Placebo & NA & 39 & $2.45 /$ week & 4 weeks & \\
\hline Emmanuel AV, & $\mathrm{CC}$ & Arm 1 & Prucalopride & $1 \mathrm{mg}$ & 37 & $1.8 /$ week & 4 weeks & 1 \\
\hline et al. [45] & & Arm 2 & Placebo & NA & 36 & $-0.7 /$ week & 4 weeks & \\
\hline Fukudo S, et al. & $\mathrm{CC}$ & Arm 1 & Lubiprostone & $48 \mu \mathrm{g}$ & 62 & 2.74/week & 2 weeks & 0 \\
\hline$[46]$ & & Arm 2 & Placebo & $\mathrm{NA}$ & 62 & $1.33 /$ week & 2 weeks & \\
\hline Irving $\mathrm{G}$, et al. & OIC & Arm 1 & Alvimopan & $0.5 \mathrm{mg}$ & 161 & $3.19 /$ week & 12 weeks & 0 \\
\hline$[47]$ & & Arm 2 & Alvimopan & $1 \mathrm{mg}$ & 160 & $3.05 /$ week & 12 weeks & \\
\hline & & Arm 3 & Placebo & NA & 164 & 2.18/week & 12 weeks & \\
\hline Jamal MM, et al. & OIC & Arm 1 & Lubiprostone & $48 \mu \mathrm{g}$ & 212 & $3.2 /$ week & 12 weeks & 0 \\
\hline$[48]$ & & Arm 2 & Placebo & NA & 212 & 2.4/week & 12 weeks & \\
\hline Jansen JP, et al. & OIC & Arm 1 & Alvimopan & $0.5 \mathrm{mg}$ & 174 & $3.42 /$ week & 12 weeks & 0 \\
\hline [49] & & Arm 2 & Alvimopan & $1 \mathrm{mg}$ & 172 & $3.51 /$ week & 12 weeks & \\
\hline & & Arm 3 & Placebo & NA & 172 & 2.01/week & 12 weeks & \\
\hline Johanson JF, et al. & $\mathrm{CC}$ & Arm 1 & Lubiprostone & $48 \mu \mathrm{g}$ & 120 & $3.69 /$ week & 2 weeks & 0 \\
\hline$[50]$ & & Arm 2 & Placebo & $\mathrm{NA}$ & 122 & $1.71 /$ week & 2 weeks & \\
\hline Johanson JF, et al. & $\mathrm{CC}$ & Arm 1 & Tegaserod & $4 \mathrm{mg}$ & 450 & $1.9 /$ week & 12 weeks & 1 \\
\hline$[51]$ & & Arm 2 & Tegaserod & $12 \mathrm{mg}$ & 451 & 1.9/week & 12 weeks & \\
\hline & & Arm 3 & Placebo & NA & 447 & $0.9 /$ week & 12 weeks & \\
\hline Johnston JM, & $\mathrm{CC}$ & Arm 1 & Linaclotide & $100 \mu \mathrm{g}$ & 12 & 6.18/week & 2 weeks & $\mathrm{N}$ \\
\hline et al. [52] & & Arm 2 & Placebo & & 10 & 2.76/week & 2 weeks & \\
\hline
\end{tabular}


Table 1 (continued)

\begin{tabular}{|c|c|c|c|c|c|c|c|c|}
\hline Reference & Disease & Arm & Drug & $\begin{array}{l}\text { Dose/ } \\
\text { day }\end{array}$ & Patient number & $\begin{array}{l}\text { Change from } \\
\text { baseline in } \mathrm{SBM}^{\mathrm{a}}\end{array}$ & $\begin{array}{l}\text { Time point of } \\
\text { extracted data }\end{array}$ & $\begin{array}{l}\text { Baseline weekly } \\
\operatorname{SBM}^{\mathrm{b}}(\geq 3: 1 ;<3: 0 \\
\text { unknown: } \mathrm{N})\end{array}$ \\
\hline \multirow{5}{*}{$\begin{array}{l}\text { Johnston JM, } \\
\text { et al. [53] }\end{array}$} & \multirow[t]{5}{*}{ IBS-C } & Arm 1 & Linaclotide & $75 \mu \mathrm{g}$ & 79 & 4.62/week & 12 weeks & \multirow[t]{5}{*}{ Differ by groups } \\
\hline & & Arm 2 & Linaclotide & $150 \mu \mathrm{g}$ & 82 & 4.36/week & 12 weeks & \\
\hline & & Arm 3 & Linaclotide & $300 \mu \mathrm{g}$ & 84 & 4.97/week & 12 weeks & \\
\hline & & Arm 4 & Linaclotide & $600 \mu \mathrm{g}$ & 89 & 5.64/week & 12 weeks & \\
\hline & & Arm 5 & Placebo & NA & 85 & $1.68 /$ week & 12 weeks & \\
\hline \multirow{2}{*}{$\underset{[54]}{\text { Kamm MA, et al. }}$} & \multirow[t]{2}{*}{$\mathrm{CC}$} & Arm 1 & Placebo & NA & 121 & $0.8 /$ week & 4 weeks & \multirow[t]{2}{*}{1} \\
\hline & & Arm 2 & Bisacodyl & $10 \mathrm{mg}$ & 247 & 5.4/week & 4 weeks & \\
\hline \multirow{3}{*}{$\begin{array}{l}\text { Kamm MA, et al. } \\
{[55]}\end{array}$} & \multirow[t]{3}{*}{$\mathrm{CC}$} & Arm 1 & Tegaserod & $4 \mathrm{mg}$ & 417 & 1.6/week & 12 weeks & \multirow[t]{3}{*}{1} \\
\hline & & Arm 2 & Tegaserod & $12 \mathrm{mg}$ & 431 & 2/week & 12 weeks & \\
\hline & & Arm 3 & Placebo & NA & 416 & 0.9/week & 12 weeks & \\
\hline \multirow{2}{*}{$\begin{array}{l}\text { Kienzle-Horn S, } \\
\text { et al. [56] }\end{array}$} & \multirow[t]{2}{*}{$\mathrm{CC}$} & Arm 1 & Bisacodyl & $10 \mathrm{mg}$ & 27 & $1.13 /$ day & 3 days & \multirow[t]{2}{*}{1} \\
\hline & & Arm 2 & Placebo & NA & 27 & $0.28 /$ day & 3 days & \\
\hline \multirow{3}{*}{$\begin{array}{l}\text { Lacy BE, et al. } \\
{[57]}\end{array}$} & \multirow[t]{3}{*}{$\mathrm{CC}$} & Arm 1 & Linaclotide & $145 \mu \mathrm{g}$ & 153 & $3.5 /$ week & 12 weeks & \multirow[t]{3}{*}{0} \\
\hline & & Arm 2 & Linaclotide & $290 \mu \mathrm{g}$ & 159 & 3.6/week & 12 weeks & \\
\hline & & Arm 3 & Placebo & NA & 171 & $1.5 /$ week & 12 weeks & \\
\hline \multirow{5}{*}{$\begin{array}{l}\text { Lembo AJ, et al. } \\
\text { [58] }\end{array}$} & \multirow[t]{5}{*}{$\mathrm{CC}$} & Arm 1 & Linaclotide & $75 \mu \mathrm{g}$ & 59 & 2.6/week & 4 weeks & \multirow[t]{5}{*}{0} \\
\hline & & Arm 2 & Linaclotide & $150 \mu \mathrm{g}$ & 56 & 3.3/week & 4 weeks & \\
\hline & & Arm 3 & Linaclotide & $300 \mu \mathrm{g}$ & 62 & 3.6/week & 4 weeks & \\
\hline & & Arm 4 & Linaclotide & $600 \mu \mathrm{g}$ & 62 & 4.3/week & 4 weeks & \\
\hline & & Arm 5 & Placebo & NA & 68 & $1.5 /$ week & 4 weeks & \\
\hline \multirow{3}{*}{$\begin{array}{l}\text { Lembo AJ, et al. } \\
\text { [59] }\end{array}$} & \multirow[t]{3}{*}{$\mathrm{CC}$} & Arm 1 & Linaclotide & $145 \mu \mathrm{g}$ & 217 & 3/week & 12 weeks & 0 \\
\hline & & Arm 2 & Linaclotide & $290 \mu \mathrm{g}$ & 216 & $3 /$ week & 12 weeks & \\
\hline & & Arm 3 & Placebo & NA & 209 & 1.1/week & 12 weeks & \\
\hline Lembo AJ, et al. & $\mathrm{CC}$ & Arm 1 & Linaclotide & $145 \mu \mathrm{g}$ & 213 & 3.4/week & 12 weeks & 0 \\
\hline [59] & & Arm 2 & Linaclotide & $290 \mu \mathrm{g}$ & 202 & 3.7/week & 12 weeks & \\
\hline & & Arm 3 & Placebo & NA & 215 & 1.1/week & 12 weeks & \\
\hline Heitland W, et al. & $\mathrm{CC}$ & Arm 1 & Lactulose & $20 \mathrm{~g}$ & 20 & $0.36 /$ day & 2 weeks & Differ by groups \\
\hline$[60]$ & & Arm 2 & Lactitol & $20 \mathrm{~g}$ & 30 & $0.49 /$ day & 2 weeks & \\
\hline Hongo M, et al. & $\mathrm{CC}$ & Arm 1 & Lubiprostone & $16 \mu \mathrm{g}$ & 41 & 2.3/week & 1 week & $\mathrm{N}$ \\
\hline [61] & & Arm 2 & Lubiprostone & $32 \mu \mathrm{g}$ & 43 & 3.5/week & 1 week & \\
\hline & & Arm 3 & Lubiprostone & $48 \mu \mathrm{g}$ & 44 & 6.8/week & 1 week & \\
\hline & & Arm 4 & Placebo & NA & 42 & $1.5 /$ week & 1 week & \\
\hline Sanders M, et al. & OIC & Arm 1 & Naloxone & $2.5 \mathrm{mg}$ & 8 & $2.21 /$ week & 3 weeks & 0 \\
\hline$[62]$ & & Arm 2 & Naloxone & $5 \mathrm{mg}$ & 8 & 2.36/week & 3 weeks & \\
\hline & & Arm 3 & Naloxone & $10 \mathrm{mg}$ & 8 & 4.1/week & 3 weeks & \\
\hline & & Arm 4 & Naloxone & $20 \mathrm{mg}$ & 7 & 5.19/week & 3 weeks & \\
\hline & & Arm 5 & Placebo & NA & 8 & $1.38 /$ week & 3 weeks & \\
\hline Shroff S, et al. & $\mathrm{CC}$ & Arm 1 & Lubiprostone & $48 \mu \mathrm{g}$ & 34 & 4.12/week & 4 weeks & $\mathrm{N}$ \\
\hline$[63]$ & & Arm 2 & Placebo & NA & 36 & 2.48/week & 4 weeks & \\
\hline Sloots CE, et al. & OIC & Arm 1 & Placebo & NA & 66 & $1.5 /$ week & 4 weeks & 0 \\
\hline [64] & & Arm 2 & Prucalopride & $2 \mathrm{mg}$ & 66 & 2.2/week & 4 weeks & \\
\hline & & Arm 3 & Prucalopride & $4 \mathrm{mg}$ & 64 & $2.5 /$ week & 4 weeks & \\
\hline Sobhani I, et al. & $\mathrm{CC}$ & Arm 1 & Lactulose & $10 \mathrm{~g}$ & 99 & $5.09 /$ week & 3 weeks & 0 \\
\hline$[65]$ & & Arm 2 & Lactulose & $20 \mathrm{~g}$ & 99 & 4.88/week & 3 weeks & \\
\hline Tomás-Ridocci & $\mathrm{CC}$ & Arm 1 & Ispaghula & $20 \mathrm{~g}$ & 10 & 5.5/week & 4 weeks & 0 \\
\hline M, et al. [66] & & Arm 2 & Placebo & NA & 10 & $1.15 /$ week & 4 weeks & \\
\hline & OIC & Arm 1 & Naloxegol & $5 \mathrm{mg}$ & 31 & $1.3 /$ week & 4 weeks & 0 \\
\hline & & Arm 2 & Naloxegol & $25 \mathrm{mg}$ & 29 & 3/week & 4 weeks & \\
\hline
\end{tabular}


Table 1 (continued)

\begin{tabular}{|c|c|c|c|c|c|c|c|c|}
\hline Reference & Disease & Arm & Drug & $\begin{array}{l}\text { Dose/ } \\
\text { day }\end{array}$ & Patient number & $\begin{array}{l}\text { Change from } \\
\text { baseline in } \mathrm{SBM}^{\mathrm{a}}\end{array}$ & $\begin{array}{l}\text { Time point of } \\
\text { extracted data }\end{array}$ & $\begin{array}{l}\text { Baseline weekly } \\
\operatorname{SBM}^{\mathrm{b}}(\geq 3: 1 ;<3: 0 \\
\text { unknown: } \mathrm{N})\end{array}$ \\
\hline \multirow{2}{*}{$\begin{array}{l}\text { Webster L, et al. } \\
\text { [67] }\end{array}$} & & Arm 3 & Naloxegol & $50 \mathrm{mg}$ & 30 & $3.5 /$ week & 4 weeks & \\
\hline & & Arm 4 & Placebo & NA & 95 & $1.3 /$ week & 4 weeks & \\
\hline \multirow[t]{2}{*}{ Xu Z, et al. [68] } & $\mathrm{CC}$ & Arm 1 & Lactitol & $10 \mathrm{~g}$ & 63 & 4.29/week & 7 days & 0 \\
\hline & & Arm 2 & Lactulose & $10 \mathrm{~g}$ & 66 & 4.29/week & 7 days & \\
\hline \multirow{2}{*}{$\begin{array}{l}\text { Fenn GC, et al. } \\
\text { [69] }\end{array}$} & $\mathrm{CC}$ & Arm 1 & Ispaghula & $10.8 \mathrm{~g}$ & 91 & 4.7/week & 14 days & 0 \\
\hline & & Arm 2 & Placebo & NA & 84 & $2.2 /$ week & 14 days & \\
\hline \multirow[t]{2}{*}{ Lin SR, et al. [70] } & $\mathrm{CC}$ & Arm 1 & Tegaserod & $12 \mathrm{mg}$ & 304 & $1.57 /$ week & 4 weeks & $\mathrm{N}$ \\
\hline & & Arm 2 & Placebo & & 303 & $0.89 /$ week & 4 weeks & \\
\hline \multirow{2}{*}{$\begin{array}{l}\text { Mareya S, et al. } \\
{[71]}\end{array}$} & OIC & Arm 1 & Lubiprostone & $48 \mu \mathrm{g}$ & 572 & $3.2 /$ week & 12 weeks & $\mathrm{N}$ \\
\hline & & Arm 2 & Placebo & NA & 568 & $2.7 /$ week & 12 weeks & \\
\hline \multirow{3}{*}{$\begin{array}{l}\text { Miner PB, et al. } \\
\text { [72] }\end{array}$} & $\mathrm{CC}$ & Arm 1 & Plecanatide & $3 \mathrm{mg}$ & 453 & $3.2 /$ week & 12 weeks & 0 \\
\hline & & Arm 2 & Plecanatide & $6 \mathrm{mg}$ & 441 & 3.1/week & 12 weeks & \\
\hline & & Arm 3 & Placebo & NA & 452 & $1.3 /$ week & 12 weeks & \\
\hline \multirow{4}{*}{$\begin{array}{l}\text { Müller-Lissner S, } \\
\text { et al. [73] }\end{array}$} & $\mathrm{CC}$ & Arm 1 & Prucalopride & $1 \mathrm{mg}$ & 76 & 2.4/week & 4 weeks & 1 \\
\hline & & Arm 2 & Prucalopride & $2 \mathrm{mg}$ & 75 & 1.9/week & 4 weeks & \\
\hline & & Arm 3 & Prucalopride & $4 \mathrm{mg}$ & 79 & $1.9 /$ week & 4 weeks & \\
\hline & & Arm 4 & Placebo & NA & 70 & $0.9 /$ week & 4 weeks & \\
\hline \multirow{2}{*}{$\begin{array}{l}\text { Novick J, et al. } \\
\text { [74] }\end{array}$} & IBS-C & Arm 1 & Tegaserod & $12 \mathrm{mg}$ & 767 & $2.45 /$ week & 12 weeks & 1 \\
\hline & & Arm 2 & Placebo & NA & 752 & $1.65 /$ week & 12 weeks & \\
\hline \multirow{3}{*}{$\begin{array}{l}\text { Paulson DM, } \\
\text { et al. [75] }\end{array}$} & OIC & Arm 1 & Alvimopan & $0.5 \mathrm{mg}$ & 58 & 1.6/week & 3 weeks & 1 \\
\hline & & Arm 2 & Alvimopan & $1 \mathrm{mg}$ & 56 & 2.9/week & 3 weeks & \\
\hline & & Arm 3 & Placebo & NA & 54 & $1.2 /$ week & 3 weeks & \\
\hline \multirow{4}{*}{$\begin{array}{l}\text { Rauck R, et al. } \\
{[76]}\end{array}$} & OIC & Arm 1 & Methylnaltrexone & $150 \mathrm{mg}$ & 201 & 2/week & 12 weeks & 0 \\
\hline & & Arm 2 & Methylnaltrexone & $300 \mathrm{mg}$ & 201 & 2.4/week & 12 weeks & \\
\hline & & Arm 3 & Methylnaltrexone & $450 \mathrm{mg}$ & 200 & 2.4/week & 12 weeks & \\
\hline & & Arm 4 & Placebo & NA & 201 & 1.9/week & 12 weeks & \\
\hline \multirow{2}{*}{$\begin{array}{l}\text { NCT02291679 } \\
\quad[77]\end{array}$} & $\mathrm{CC}$ & Arm 1 & Linaclotide & $72 \mu \mathrm{g}$ & 411 & 2.366/week & 12 weeks & 0 \\
\hline & & Arm 2 & Placebo & NA & 401 & $1.329 /$ week & 12 weeks & \\
\hline \multirow{5}{*}{$\begin{array}{l}\text { NCT00402337 } \\
\quad[78]\end{array}$} & $\mathrm{CC}$ & Arm 1 & Linaclotide & $72 \mu \mathrm{g}$ & 54 & 2.59/week & 4 weeks & $\mathrm{N}$ \\
\hline & & Arm 2 & Linaclotide & $145 \mu \mathrm{g}$ & 51 & $3.25 /$ week & 4 weeks & \\
\hline & & Arm 3 & Linaclotide & $290 \mu \mathrm{g}$ & 58 & 3.57/week & 4 weeks & \\
\hline & & Arm 4 & Linaclotide & $579 \mu \mathrm{g}$ & 51 & 4.29/week & 4 weeks & \\
\hline & & Arm 5 & Placebo & NA & 61 & $1.45 /$ week & 4 weeks & \\
\hline \multirow{2}{*}{$\begin{array}{l}\text { NCT00948818 } \\
\text { [79] }\end{array}$} & IBS-C & Arm 1 & Linaclotide & $290 \mu \mathrm{g}$ & 405 & 3.898/week & 12 weeks & $\mathrm{N}$ \\
\hline & & Arm 2 & Placebo & NA & 395 & 1.13/week & 12 weeks & \\
\hline \multirow{2}{*}{$\begin{array}{l}\text { NCT01880424 } \\
\quad[80]\end{array}$} & IBS-C & Arm 1 & Linaclotide & $290 \mu \mathrm{g}$ & 417 & 2.96/week & 12 weeks & $\mathrm{N}$ \\
\hline & & Arm 2 & Placebo & NA & 422 & $1.51 /$ week & 12 weeks & \\
\hline \multirow{2}{*}{$\begin{array}{l}\text { NCT00380250 } \\
\quad[81]\end{array}$} & IBS-C & Arm 1 & Lubiprostone & $16 \mu \mathrm{g}$ & 390 & $1.59 /$ week & 2 months & $\mathrm{N}$ \\
\hline & & Arm 2 & Placebo & NA & 193 & $1.41 /$ week & 2 months & \\
\hline \multirow{5}{*}{$\begin{array}{l}\text { Fukudo S, et al. } \\
\text { [33] }\end{array}$} & IBS-C & Arm 1 & Linaclotide & $62.5 \mu \mathrm{g}$ & 103 & 2.81/week & 2 months & 0 \\
\hline & & Arm 2 & Linaclotide & $125 \mu \mathrm{g}$ & 103 & $3.43 /$ week & 2 months & \\
\hline & & Arm 3 & Linaclotide & $250 \mu \mathrm{g}$ & 103 & $3.15 /$ week & 2 months & \\
\hline & & Arm 4 & Linaclotide & $500 \mu \mathrm{g}$ & 98 & 3.11/week & 2 months & \\
\hline & & Arm 5 & Placebo & NA & 103 & $1.77 /$ week & 2 months & \\
\hline \multirow{3}{*}{$\begin{array}{l}\text { Fukudo S, et al. } \\
\text { [32] }\end{array}$} & $\mathrm{CC}$ & Arm 1 & Linaclotide & $62.5 \mu \mathrm{g}$ & 78 & $3.47 /$ week & 2 weeks & 0 \\
\hline & & Arm 2 & Linaclotide & $125 \mu \mathrm{g}$ & 69 & 2.86/week & 2 weeks & \\
\hline & & Arm 3 & Linaclotide & $250 \mu \mathrm{g}$ & 72 & 3.73/week & 2 weeks & \\
\hline
\end{tabular}


Table 1 (continued)

\begin{tabular}{|c|c|c|c|c|c|c|c|c|}
\hline Reference & Disease & Arm & Drug & $\begin{array}{l}\text { Dose/ } \\
\text { day }\end{array}$ & Patient number & $\begin{array}{l}\text { Change from } \\
\text { baseline in } \mathrm{SBM}^{\mathrm{a}}\end{array}$ & $\begin{array}{l}\text { Time point of } \\
\text { extracted data }\end{array}$ & $\begin{array}{l}\text { Baseline weekly } \\
\operatorname{SBM}^{\mathrm{b}}(\geq 3: 1 ;<3: 0 ; \\
\text { unknown: } \mathrm{N})\end{array}$ \\
\hline & & Arm 4 & Linaclotide & $500 \mu \mathrm{g}$ & 74 & 3.97/week & 2 weeks & \\
\hline & & Arm 5 & Placebo & NA & 80 & $1.53 /$ week & 2 weeks & \\
\hline \multirow{2}{*}{$\begin{array}{l}\text { Fukudo S, et al. } \\
\text { [30] }\end{array}$} & \multirow[t]{2}{*}{ IBS-C } & Arm 1 & Linaclotide & $500 \mu \mathrm{g}$ & 249 & $3.14 /$ week & 12 weeks & \multirow[t]{2}{*}{0} \\
\hline & & Arm 2 & Placebo & NA & 251 & $1.49 /$ week & 12 weeks & \\
\hline \multirow{2}{*}{$\begin{array}{l}\text { Fukudo S, et al. } \\
\text { [31] }\end{array}$} & \multirow[t]{2}{*}{$\mathrm{CC}$} & Arm 1 & Linaclotide & $500 \mu \mathrm{g}$ & 90 & 3.78/week & 2 weeks & \multirow[t]{2}{*}{0} \\
\hline & & Arm 2 & Placebo & NA & 88 & $1.25 /$ week & 2 weeks & \\
\hline
\end{tabular}

$C C$ chronic constipation, $I B S-C$ irritable bowel syndrome with constipation, $N A$ not applicable, $O I C$ opioid-induced constipation, $S B M$ spontaneous bowel movement

${ }^{a}$ Change from baseline in daily or weekly number of SBM after treatment

${ }^{\mathrm{b}}$ Weekly SBM of enrolled patients at the start of each respective trial

71], and baseline SBM values were unknown in 9 studies [52, $61,63,70,71,78-81]$. The distribution of male and female patients across the included studies was non-uniform, with significantly more female patients than male patients in most studies (Table S2). The average treatment duration across the trials ranged from 3 days to 12 weeks (Table 1).

\section{Treatment Network}

A total of 47 treatments for 16 constipation oral drugs was plotted in the network for the primary analysis (Fig. 3). For all treatment arms, placebo was a common reference comparator treatment arm. The most frequently studied agents were linaclotide (trials $=16$, patients $=$ 4656) and lubiprostone (trials $=9$, patients $=1674$ ). The most frequently used comparisons were linaclotide versus placebo (trials $=16$ ) and lubiprostone versus placebo (trials $=9$ ). There were 3 direct head-to-head comparisons between 2 active treatments (lactulose $20 \mathrm{~g}$ versus lactitol $20 \mathrm{~g}$, lactulose $10 \mathrm{~g}$ versus lactitol $10 \mathrm{~g}$, and PEG $20 \mathrm{~g}$ versus lactulose $20 \mathrm{~g}$ ) and 25 comparisons between different dosages of the same treatment (Table 1 and Fig. 3). Each treatment in the NMA pooled data was from trials of different durations. There were no significant inconsistencies for the indirect evidence within the NMA. Therefore, a consistency model was applied for the NMA.

\section{NMA Results}

Indirect comparison of the 47 selected constipation treatments with linaclotide $500 \mu \mathrm{g}$ showed that linaclotide $500 \mu \mathrm{g}$ was more effective in terms of the change in weekly SBM before and after treatment than most other treatments (Table 2 and Fig. 4). When the mean difference in the change in weekly SBM with other constipation treatments was compared with that of linaclotide $500 \mu \mathrm{g}$, linaclotide $500 \mu \mathrm{g}$ was statistically significantly more effective than placebo $(-1.907 ;-2.568$ to $-1.237)$; lubiprostone $16 \mu \mathrm{g}(-2.090 ;-3.226$ to -0.968$)$; methylnaltrexone $150 \mathrm{mg}(-1.807 ;-3.126$ to -0.491$)$, $300 \mathrm{mg}(-1.411 ;-2.722$ to -0.096$)$, and $450 \mathrm{mg}(-1.405$; -2.708 to -0.097$)$; naloxegol $5 \mathrm{mg}(-2.074 ;-4.001$ to $-0.131)$ and $12.5 \mathrm{mg}(-1.329 ;-2.347$ to -0.318$)$; tegaserod $4 \mathrm{mg}(-1.133 ;-2.059$ to -0.207$)$; and tegaserod $12 \mathrm{mg}(-1.024 ;-1.822$ to -0.228$)$. Linaclotide $500 \mu \mathrm{g}$ was statistically significantly less
Fig. 2 Overall risk of bias across all selected studies

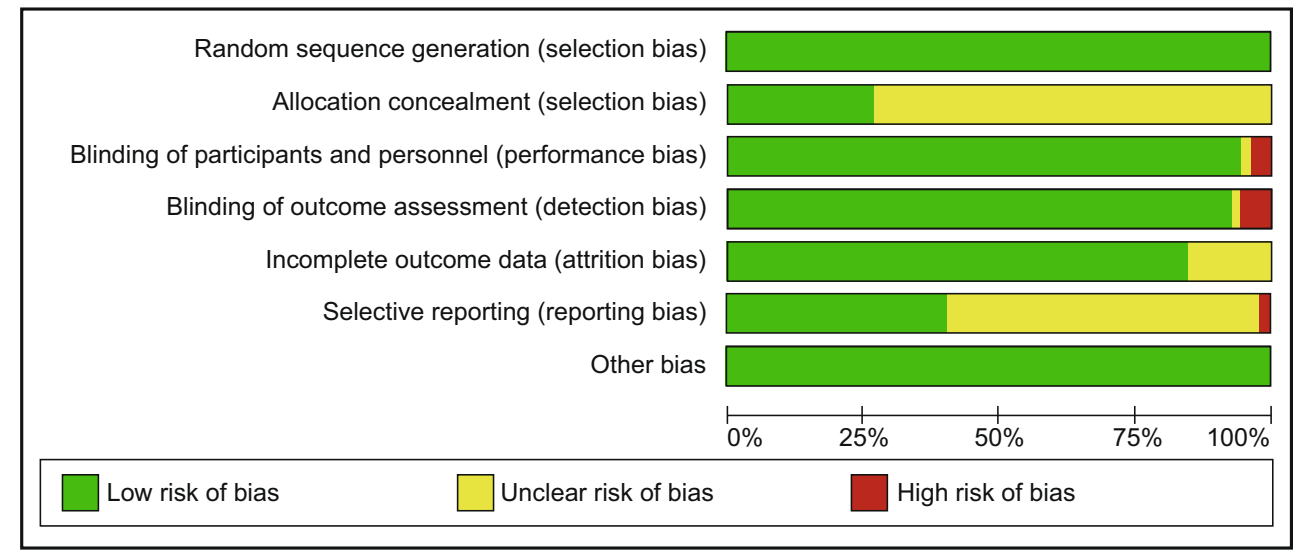


Fig. 3 Treatment network for the network meta-analysis. The size of each node represents the number of patients pooled for each treatment and the thickness of the edges represents the number of trials for each comparison. Altogether, 47 treatments/16 drugs were included in the NMA. Different doses of the same drug were treated as separate treatments. Clinically equivalent doses of the same treatment were pooled together. $\mathrm{CC}$, chronic constipation; IBS-C, irritable bowel syndrome with constipation; NMA, network meta-analysis; OIC, opioidinduced constipation; PEG, polyethylene glycol

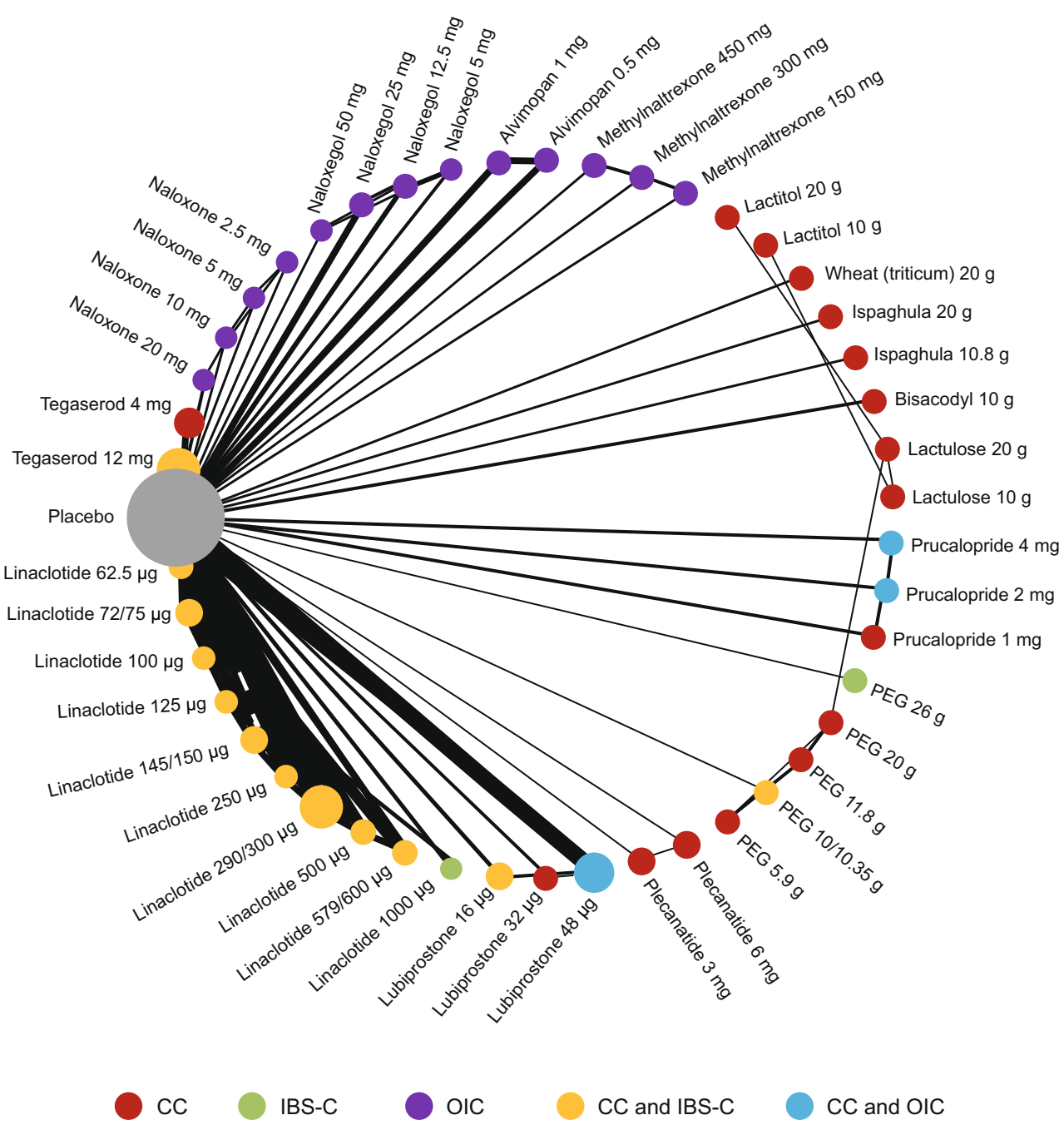

effective than the non-approved dose of linaclotide $600 \mu \mathrm{g}(1.159 ; 0.123$ to 2.199$)$ and bisacodyl $10 \mathrm{mg}$ $(2.979 ; 1.723$ to 4.233$)$. The differences in efficacy between linaclotide $500 \mu \mathrm{g}$ and other doses of linaclotide were not statistically significant.

\section{Sensitivity Analysis Results}

Sensitivity analyses were conducted on trials involving only CC (26 trials; Fig. 5a), trials with only CC and IBS-C (43 trials; Fig. 5b), trials with only severe constipation (30 trials; Fig. 6a), and trials with only a low risk of bias (48 trials; Fig. $6 \mathrm{~b})$. The effects of linaclotide $500 \mu \mathrm{g}$ were mostly consistent across each of the sensitivity analyses conducted. Linaclotide $500 \mu \mathrm{g}$ remained significantly more effective than each of the comparators identified in the primary analysis and was significantly less effective than bisacodyl $10 \mathrm{mg}$. Additionally, linaclotide $500 \mu \mathrm{g}$ was significantly more effective than lubiprostone $16 \mu \mathrm{g}$ when the sensitivity analysis included trials involving only CC (Fig. 5a), trials involving CC and IBS-
C (Fig. 5b), and trials with a low risk of bias (Fig. 6b). However, the sensitivity analysis of trials involving only $\mathrm{CC}$ showed that there was no significant difference between linaclotide $500 \mu \mathrm{g}$ and the non-approved dose of linaclotide $600 \mu \mathrm{g}$ (Fig. 5a), which is in contrast to the findings from the primary analysis (Fig. 4).

\section{Discussion}

This is the first study to compare the approved standard dose of linaclotide $500 \mu \mathrm{g}$ in Japan with other constipation treatments available worldwide. While lower doses of linaclotide are approved for CC $(72 \mu \mathrm{g}, 145 \mu \mathrm{g})$ in the US and IBS-C $(290 \mu \mathrm{g})$ in the US and European Union [14, 82], doseranging studies conducted in Japan showed that the $500-\mu \mathrm{g}$ dose was the optimal dose in this population in terms of efficacy and safety $[30,32,33]$. The reasons for the higher dose of linaclotide in Japan may be due to a weaker responsiveness to linaclotide in Japanese patients than in Western patients due to 
Table 2 Efficacy of constipation treatments in terms of mean change from baseline in weekly SBM in relation to linaclotide $500 \mu \mathrm{g}$

\begin{tabular}{|c|c|c|c|c|c|}
\hline \multirow{2}{*}{$\begin{array}{l}\text { Treatment } \\
\text { Linaclotide } 500 \mu \mathrm{g}\end{array}$} & \multirow{2}{*}{$\begin{array}{l}\text { Number of patients } \\
511\end{array}$} & \multirow{2}{*}{$\begin{array}{l}\text { Treatment durations } \\
\text { in included studies }\end{array}$} & \multirow{2}{*}{$\begin{array}{l}\text { Mean difference from } \\
\text { linaclotide } 500 \mu \mathrm{g}\end{array}$} & \multicolumn{2}{|c|}{$95 \%$ credible interval } \\
\hline & & & & 0 & 0 \\
\hline Placebo & 8554 & & -1.907 & -2.568 & -1.237 \\
\hline Linaclotide $62.5 \mu \mathrm{g}$ & 181 & 2 weeks, 2 months & -0.420 & -1.383 & 0.538 \\
\hline Linaclotide $72 / 75 \mu \mathrm{g}$ & 603 & 4 weeks, 12 weeks & -0.481 & -1.385 & 0.424 \\
\hline Linaclotide $100 \mu \mathrm{g}$ & 24 & 5 days, 2 weeks & 0.835 & -1.789 & 3.485 \\
\hline Linaclotide $125 \mu \mathrm{g}$ & 172 & 2 weeks, 2 months & -0.337 & -1.310 & 0.645 \\
\hline Linaclotide $145 / 150 \mu \mathrm{g}$ & 772 & 4 weeks, 12 weeks & 0.115 & -0.720 & 0.949 \\
\hline Linaclotide $250 \mu \mathrm{g}$ & 175 & 2 weeks, 2 months & -0.120 & -1.094 & 0.857 \\
\hline Linaclotide $290 / 300 \mu \mathrm{g}$ & 2004 & 4 weeks, 12 weeks & 0.363 & -0.400 & 1.136 \\
\hline Linaclotide $579 / 600 \mu \mathrm{g}$ & 202 & 4 weeks, 12 weeks & 1.159 & 0.123 & 2.199 \\
\hline Linaclotide $1000 \mu \mathrm{g}$ & 12 & 5 days & 3.181 & -0.175 & 6.537 \\
\hline Lubiprostone $16 \mu \mathrm{g}$ & 431 & 1 week, 2 months & -2.090 & -3.226 & -0.968 \\
\hline Lubiprostone $32 \mu \mathrm{g}$ & 43 & 1 week & -1.539 & -3.311 & 0.228 \\
\hline Lubiprostone $48 \mu \mathrm{g}$ & 1200 & 1 week, 2 weeks, 4 weeks, 12 weeks & -0.431 & -1.278 & 0.450 \\
\hline Plecanatide $3 \mathrm{mg}$ & 453 & 12 weeks & 0.000 & -1.173 & 1.176 \\
\hline Plecanatide $6 \mathrm{mg}$ & 441 & 12 weeks & -0.096 & -1.275 & 1.077 \\
\hline PEG $5.9 \mathrm{~g}$ & 67 & 4 weeks & -1.686 & -4.826 & 1.462 \\
\hline PEG 10/10.35 g & 86 & 30 days, 4 weeks & -2.267 & -4.966 & 0.438 \\
\hline PEG $11.8 \mathrm{~g}$ & 69 & 4 weeks & -0.879 & -4.000 & 2.242 \\
\hline PEG $20 \mathrm{~g}$ & 99 & 4 weeks & -1.888 & -5.008 & 1.225 \\
\hline PEG $26 \mathrm{~g}$ & 67 & 4 weeks & -0.532 & -2.265 & 1.207 \\
\hline Prucalopride $1 \mathrm{mg}$ & 113 & 4 weeks & -0.155 & -1.517 & 1.209 \\
\hline Prucalopride $2 \mathrm{mg}$ & 141 & 4 weeks & -0.963 & -2.257 & 0.329 \\
\hline Prucalopride $4 \mathrm{mg}$ & 143 & 4 weeks & -0.823 & -2.111 & 0.483 \\
\hline Lactulose $10 \mathrm{~g}$ & 165 & 7 days, 3 weeks & -3.213 & -7.287 & 0.843 \\
\hline Lactulose $20 \mathrm{~g}$ & 152 & 2 weeks, 3 weeks, 4 weeks & -3.424 & -7.259 & 0.429 \\
\hline Bisacodyl $10 \mathrm{mg}$ & 274 & 3 days, 4 weeks & 2.979 & 1.723 & 4.233 \\
\hline Ispaghula $10.8 \mathrm{~g}$ & 91 & 14 days & 0.593 & -1.037 & 2.230 \\
\hline Ispaghula $20 \mathrm{~g}$ & 10 & 4 weeks & 2.465 & -1.395 & 6.352 \\
\hline Wheat (Triticum) $20 \mathrm{~g}$ & 12 & 4 weeks & -0.589 & -4.139 & 3.023 \\
\hline Lactitol $10 \mathrm{~g}$ & 63 & 7 days & -3.210 & -7.602 & 1.198 \\
\hline Lactitol $20 \mathrm{~g}$ & 30 & 2 weeks & -2.505 & -7.091 & 2.133 \\
\hline Methylnaltrexone $150 \mathrm{mg}$ & 201 & 12 weeks & -1.807 & -3.126 & -0.491 \\
\hline Methylnaltrexone $300 \mathrm{mg}$ & 201 & 12 weeks & -1.411 & -2.722 & -0.096 \\
\hline Methylnaltrexone $450 \mathrm{mg}$ & 200 & 12 weeks & -1.405 & -2.708 & -0.097 \\
\hline Alvimopan $0.5 \mathrm{mg}$ & 393 & 3 weeks, 12 weeks & -0.843 & -1.857 & 0.168 \\
\hline Alvimopan $1 \mathrm{mg}$ & 388 & 3 weeks, 12 weeks & -0.611 & -1.620 & 0.403 \\
\hline Naloxegol $5 \mathrm{mg}$ & 31 & 4 weeks & -2.074 & -4.001 & -0.131 \\
\hline Naloxegol $12.5 \mathrm{mg}$ & 439 & 12 weeks & -1.329 & -2.347 & -0.318 \\
\hline Naloxegol $25 \mathrm{mg}$ & 467 & 4 weeks, 12 weeks & -0.787 & -1.768 & 0.200 \\
\hline Naloxegol $50 \mathrm{mg}$ & 30 & 4 weeks & 0.122 & -1.828 & 2.095 \\
\hline Naloxone $2.5 \mathrm{mg}$ & 8 & 3 weeks & -1.081 & -5.384 & 3.240 \\
\hline Naloxone $5 \mathrm{mg}$ & 8 & 3 weeks & -0.942 & -5.262 & 3.397 \\
\hline Naloxone $10 \mathrm{mg}$ & 8 & 3 weeks & 0.809 & -3.441 & 5.111 \\
\hline Naloxone $20 \mathrm{mg}$ & 7 & 3 weeks & 1.891 & -2.547 & 6.365 \\
\hline Tegaserod $4 \mathrm{mg}$ & 867 & 12 weeks & -1.133 & -2.059 & -0.207 \\
\hline Tegaserod $12 \mathrm{mg}$ & 2125 & 4 weeks, 12 weeks & -1.024 & -1.822 & -0.228 \\
\hline
\end{tabular}

PEG polyethylene glycol, SBM spontaneous bowel movement

${ }^{a}$ Different studies of a particular treatment had different treatment durations

differences in the genes encoding the guanylate cyclase-C pathway, differences in the presence of endogenous or bacterial proteases, and differences in external factors (e.g., diet) between the two populations. The findings from this NMA provide clinically relevant information on the use of linaclotide for the management of CC and IBS-C in Japan and the relative efficacy of linaclotide $500 \mu \mathrm{g}$ compared with other constipation treatments.
Findings from the SLR and NMA demonstrated that, in terms of the mean difference in the change in weekly SBM between the Japanese standard dose of linaclotide $500 \mu \mathrm{g}$ and other constipation treatments, the Japanese standard dose was significantly more effective than lubiprostone $16 \mu \mathrm{g}$; methylnaltrexone 150, 300, and $450 \mathrm{mg}$; naloxegol 5 and $12.5 \mathrm{mg}$; and tegaserod 4 and $12 \mathrm{mg}$. The standard dose was significantly less effective than the non-approved dose of 


\section{Constipation treatments}

\section{Placebo}

Linaclotide $500 \mu \mathrm{g}$

Linaclotide $62.5 \mu \mathrm{g}$

Linaclotide $72 / 75 \mu \mathrm{g}$

Linaclotide $100 \mu \mathrm{g}$

Linaclotide $125 \mu \mathrm{g}$

Linaclotide 145/150 $\mu \mathrm{g}$

Linaclotide $250 \mu \mathrm{g}$

Linaclotide $290 / 300 \mu \mathrm{g}$

Linaclotide 579/600 $\mu \mathrm{g}$

Linaclotide $1000 \mu \mathrm{g}$

Lubiprostone $16 \mu \mathrm{g}$

Lubiprostone $32 \mu \mathrm{g}$

Lubiprostone $48 \mu \mathrm{g}$

Plecanatide $3 \mathrm{mg}$

Plecanatide $6 \mathrm{mg}$

PEG $5.9 \mathrm{~g}$

PEG 10/10.35 g

PEG $11.8 \mathrm{~g}$

PEG $20 \mathrm{~g}$

PEG $26 \mathrm{~g}$

Prucalopride $1 \mathrm{mg}$

Prucalopride $2 \mathrm{mg}$

Prucalopride $4 \mathrm{mg}$

Lactulose $10 \mathrm{~g}$

Lactulose $20 \mathrm{~g}$

Bisacodyl $10 \mathrm{mg}$

Ispaghula $10.8 \mathrm{~g}$

Ispaghula $20 \mathrm{~g}$

Wheat (triticum) $20 \mathrm{~g}$

Lactitol $10 \mathrm{~g}$

Lactitol $20 \mathrm{~g}$

Methylnaltrexone $150 \mathrm{mg}$

Methylnaltrexone $300 \mathrm{mg}$

Methylnaltrexone $450 \mathrm{mg}$

Alvimopan $0.5 \mathrm{mg}$

Alvimopan $1 \mathrm{mg}$

Naloxegol $5 \mathrm{mg}$

Naloxegol $12.5 \mathrm{mg}$

Naloxegol $25 \mathrm{mg}$

Naloxegol $50 \mathrm{mg}$

Naloxone $2.5 \mathrm{mg}$

Naloxone $5 \mathrm{mg}$

Naloxone $10 \mathrm{mg}$

Naloxone $20 \mathrm{mg}$

Tegaserod $4 \mathrm{mg}$

Tegaserod $12 \mathrm{mg}$

\section{No. of patients}

8554

511

181

603

24

172
772

175

2004

202

12

431

1200

453

441

67

86
69

99

67

113

141

143

165

152

274

91

10

12

63
30
201

30
201

201

200

393

388

31

439

467

30

8

8
8

7

867

2125
Linaclotide $500 \mu \mathrm{g}$

Significantly worse than linaclotide $500 \mu \mathrm{g}^{*}$

Significantly better than linaclotide $500 \mu \mathrm{g}^{*}$

No significant difference from linaclotide $500 \mu \mathrm{g}$

Fig. 4 Overall mean difference in the number of weekly SBM before and after treatment compared with linaclotide $500 \mu \mathrm{g}$. The asterisk indicates mean differences were considered significantly different from linaclotide

linaclotide $600 \mu \mathrm{g}$ and bisacodyl $10 \mathrm{mg}$. However, interpretation of these findings should take into account that some of the treatments analyzed in the NMA included those approved for OIC (e.g., nalexegol, naloxone, alvimopan, methylnaltrexone, lubiprostone). Therefore, although our findings showed that the opioid receptor antagonists methylnaltrexone and naloxegol were less effective than linaclotide $500 \mu \mathrm{g}$, the NMA was not conducted solely in patients with OIC, and linaclotide is not an approved treatment for patients with OIC. In addition, the relatively greater efficacy of bisacodyl compared with linaclotide $500 \mu \mathrm{g}$ should take into account that the analyses only included two bisacodyl trials: one of 3 days duration [56] and the other of 4 weeks duration [54]. In comparison, the treatment durations for the four trials on linaclotide $500 \mu \mathrm{g}$ ranged from 2 to 12 weeks [30, 32, 33]. Moreover, bisacodyl, being a stimulant laxative, is more suitable for short-term use in temporary constipation and is not usually recommended in CC [83]. 
a

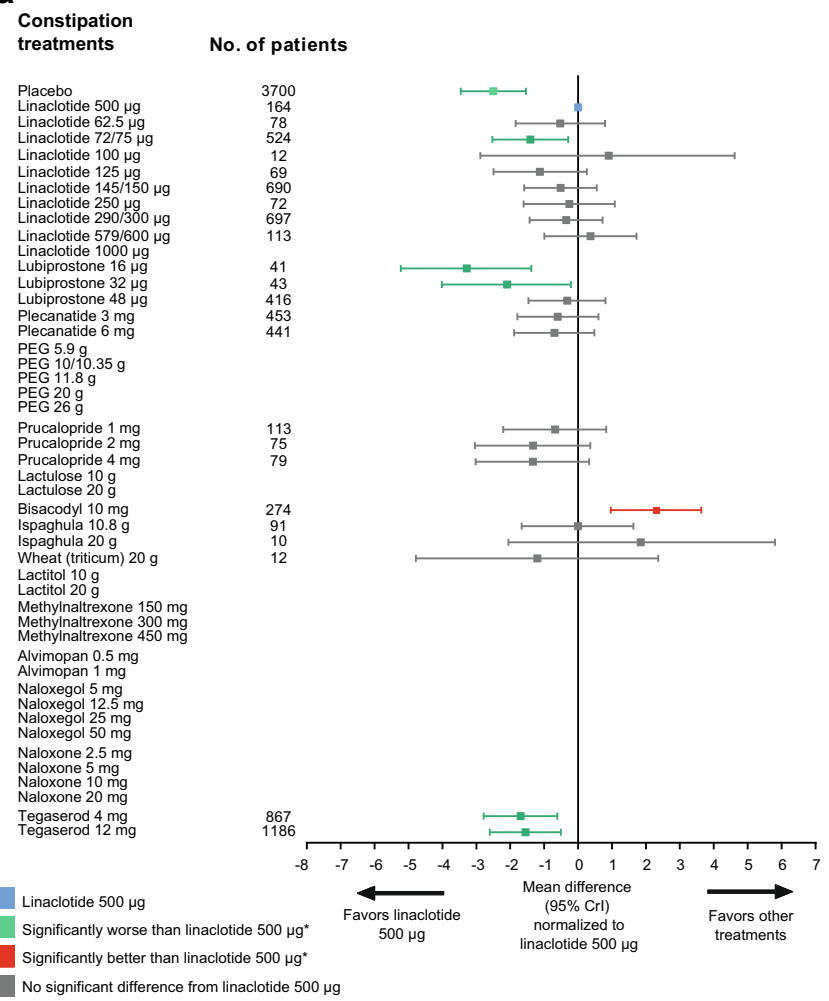

Fig. 5 Sensitivity analyses for a 26 trials of patients with only chronic constipation (i.e., 28 trials involving patients with IBS-C and OIC were excluded) and $\mathbf{b} 43$ trials of patients with only chronic constipation and IBS-C (i.e., 11 trials involving patients with OIC were excluded). Data are b

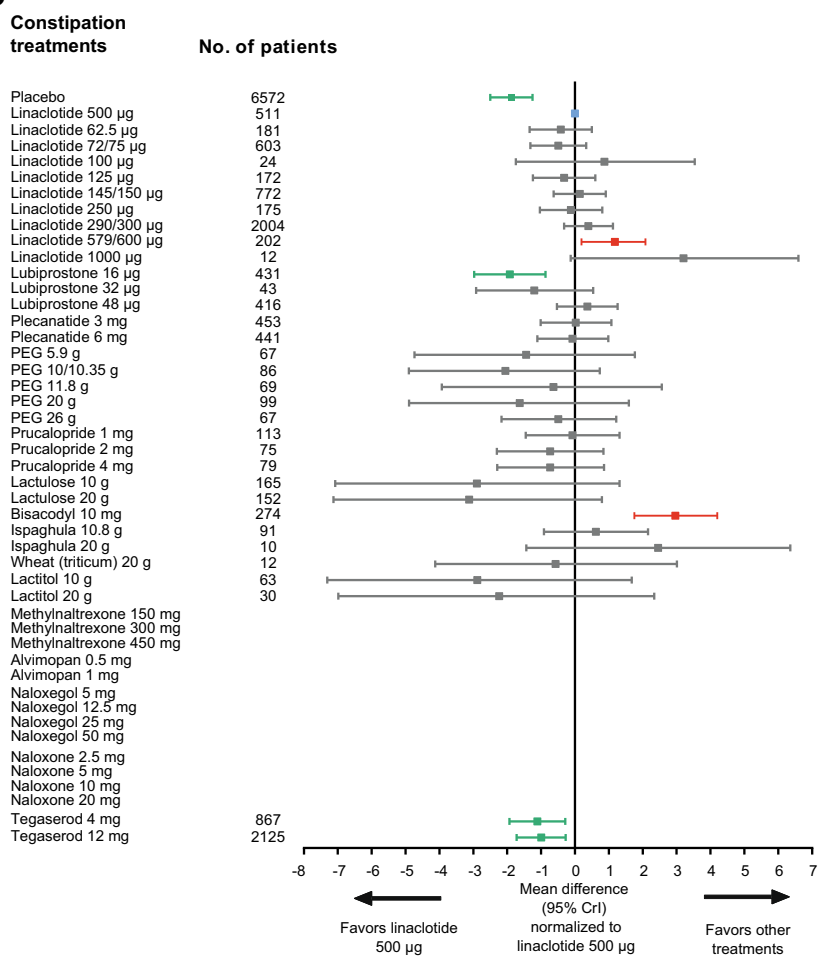

the mean difference in weekly SBM compared with linaclotide $500 \mu \mathrm{g}$. 95\% CrI, 95\% credible interval; IBS-C, irritable bowel syndrome with constipation; OIC, opioid-induced constipation; PEG, polyethylene glycol; SBM, spontaneous bowel movement

NMA was to compare all constipation treatments, including those approved for CC, IBS-C, and OIC, with the recently approved dose of linaclotide $500 \mu \mathrm{g}$ in Japan, while the Nelson study compared the efficacy between drugs for CC only and did not include patients with IBS-C or OIC. In addition, the Nelson et al. study included only 21 studies and 8 constipation treatments in the NMA, whereas the current study included 52 studies and 47 treatments (16 drugs with different dosages considered as separate treatments) in the NMA.

An additional finding from this study was that linaclotide $500 \mu \mathrm{g}$ was significantly more effective than lubiprostone $16 \mu \mathrm{g}$ (and 32 and $48 \mu \mathrm{g}$ in point estimate terms, Table 2), a chloride channel activator [84]. Lubiprostone $48 \mu \mathrm{g}$ was approved for CC in Japan in 2012 [85]. This finding suggests that linaclotide may be a suitable alternative for patients in whom lubiprostone is ineffective or contraindicated in Japanese healthcare settings. Further, in point estimate terms, linaclotide $500 \mu \mathrm{g}$ was also more effective than plecanatide 3 and $6 \mathrm{mg}$ (Table 2), which has the same pharmacological properties (guanylate cyclase-C agonistic activity) as linaclotide [86]. Together, these results suggest that head-tohead clinical trials on linaclotide active comparators, both 
a

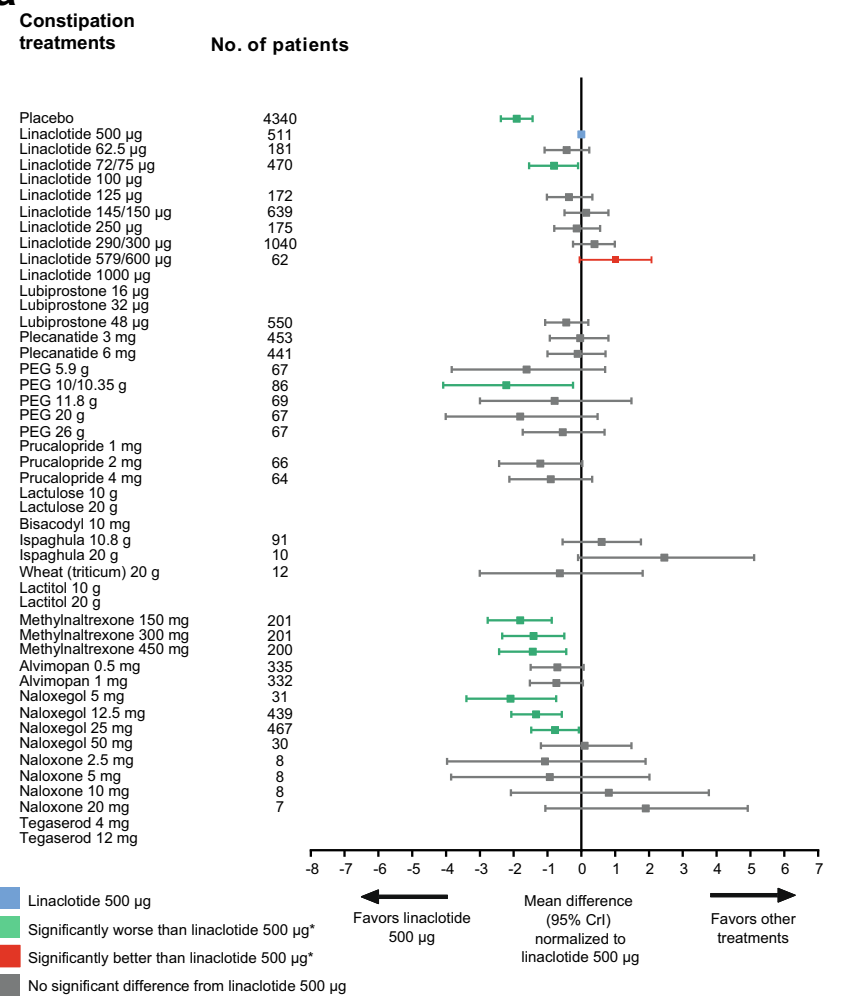

Fig. 6 Sensitivity analyses for a 30 trials of patients with average baseline $\mathrm{SBM}<3$ (i.e., 22 trials on mild-to-moderate constipation $[\geq 3 \mathrm{SBM} /$ week] and 2 trials that could not be connected in the network were excluded) and $\mathbf{b} 48$ trials without a high risk of bias (i.e., 4 trials with high

within and between drug classes, for the treatment of chronic constipation are warranted.

The main strength of this study is that it was an NMA of RCTs on constipation treatments that provides a valid statistical alternative to direct head-to-head studies [17-19]. This study used Bayesian NMA modeling, which allows for indirect comparison of treatments by combining evidence from multiple RCTs while retaining the randomization element. To minimize publication bias, both published studies and studies with results accepted for publication were included. In addition, the included studies were found to be consistent; hence, a consistency model could be applied to the NMA. By including drugs commonly used for constipation in clinical practice and different doses of the same drug as separate treatments, this NMA has provided a comprehensive analysis of the constipation treatment landscape. In addition, the inclusion of studies conducted globally and in Japan provides results that are useful for clinical treatment decisions globally and specifically in the Japanese context.

This study was subject to several limitations. First, conventional treatments such as magnesium oxide that are commonly used in Japan were not included in the NMA due to the limited amount and low quality of the available information. Additionally, constipation treatments administered rectally b

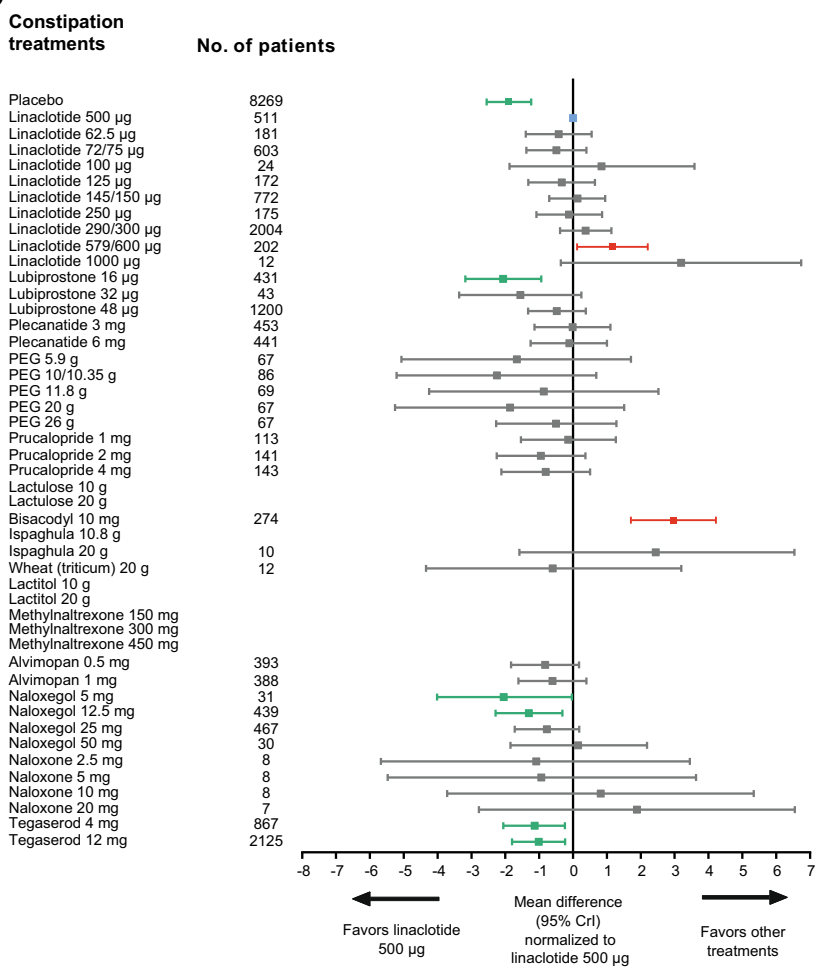

risk of bias and 2 trials that could not be connected in the network were excluded). 95\% CrI, 95\% credible interval; PEG, polyethylene glycol; SBM, spontaneous bowel movement

and newer agents in development were not included. Therefore, the influence of traditional treatments, treatments with modes of administration other than orally, and newer agents on the overall results of this study is unknown. Second, although CSBM and abdominal pain are the US Food and Drug Administration-recommended primary endpoints for assessment of constipation treatment efficacy for IBS-C [87, 88], weekly SBM was the primary endpoint for this analysis because it was the most commonly reported measure among the selected studies, which allowed us to increase the number of studies in the NMA and expand the network. Safety endpoints were also not assessed in this study. Therefore, interpretation of these results should take into consideration that assessment of only one endpoint (SBM) may not elucidate all the benefits of a particular constipation treatment, and other factors such as CSBM, abdominal symptoms, and adverse events such as diarrhea should be taken into account. Third, although linaclotide is not approved for use in OIC, studies on OIC were included in this analysis to expand the network to include all studies on target CC and IBS-C treatments that also have OIC as an approved indication (e.g., lubiprostone). Although linaclotide has been used offlabel for the treatment of OIC $[89,90]$, the use of linaclotide $500 \mu \mathrm{g}$ for patients with OIC is not currently approved or 
recommended. Fourth, this NMA featured several different doses of the included constipation treatments, as well as unapproved doses, pooling of data from different constipation indications, pooling of clinically equivalent doses, and a wide range of follow-up periods, all of which may have influenced the overall results. The criterion for statistically significant differences was based on whether the $95 \%$ CrI crossed 0 , because minimally important differences that are clinically relevant for SBM were not found in the literature. Therefore, these results should be interpreted carefully in clinical settings. Lastly, as potential heterogeneity in study designs, trial procedures, patients, and settings between the included studies may have influenced results, sensitivity analyses were conducted to address the potential effects of including different constipation types (CC, IBS-C, and OIC) and patients with different disease severities. However, other differences across the studies, including the wide range of study durations (3 days to 12 weeks) and pooling of clinically equivalent doses of linaclotide and PEG, may have contributed to the heterogeneity and should be taken into account when interpreting the results.

In conclusion, linaclotide $500 \mu \mathrm{g}$, which is the approved dose in Japan for CC and IBS-C, was found to be significantly more effective than placebo, lubiprostone $16 \mu \mathrm{g}$, and tegaserod 4 and $12 \mathrm{mg}$ and less effective than bisacodyl $10 \mathrm{mg}$ and the non-approved dose of linaclotide $600 \mu \mathrm{g}$. Linaclotide $500 \mu \mathrm{g}$ was also significantly more effective than methylnaltrexone 150,300 , and $450 \mathrm{mg}$ and naloxone $12.5 \mathrm{mg}$; however, these agents are used in OIC, which is not an approved indication for linaclotide. The results of this NMA provide relative efficacy data that are particularly useful for clinical decision-making for treatment of CC and IBS-C until head-to-head clinical trials on constipation treatments become available.

Acknowledgments We would like to thank Douglas C.A. Taylor from Ironwood Pharmaceuticals for his contribution and help in determining the clinically equivalent dosages of linaclotide for the NMA.

Role of the Sponsor Astellas Pharma Inc. was involved in the study design, data collection, data analysis, and preparation of the manuscript. Access to anonymized individual participant level data will not be provided for this study as it meets one or more of the exceptions described on www.clinicalstudydatarequest.com under "Sponsor Specific Details for Astellas."

Authors Contribution All authors participated in the interpretation of study results and in the drafting, critical revision, and approval of the final version of the manuscript. HO was involved in the study design/concept, data collection, analysis, and interpretation. WT and KI were involved in the acquisition of data and statistical analysis. SS was involved in the study design, statistical analysis, and interpretation. TO and AN were involved in the analysis and interpretation.

Funding This study was sponsored by Astellas Pharma Inc. Medical writing assistance was provided by Sandra Kurian, M. Pharm, and
Serina Stretton, PhD, CMPP, of ProScribe - Envision Pharma Group, and was funded by Astellas Pharma Inc. ProScribe's services complied with international guidelines for Good Publication Practice (GPP3, available at https://annals.org/aim/fullarticle/2424869/good-publicationpractice-communicating-company-sponsored-medical-research-gpp3).

\section{Compliance with Ethical Standards}

Conflict of Interest H. Okumura and S. Shoji are employees of Astellas Pharma Inc. K. Iwasaki is an employee of Milliman and W. Tang was an employee of Milliman at the time of the study and received funding from Astellas Pharma Inc. T. Odaka and A. Nakajima received an advisory contract fee from Astellas Pharma Inc.

Ethical Approval This study protocol was reviewed by Astellas Medical Affairs Japan Protocol Review Committee and approved. The conduct of the study was based on a protocol that has been published (Registration Number: CRD42018111737) in the PROSPERO International prospective register of systematic reviews [22].

Informed Consent Not available because this study is categorized into secondary data collection based on published data.

Open Access This article is licensed under a Creative Commons Attribution 4.0 International License, which permits use, sharing, adaptation, distribution and reproduction in any medium or format, as long as you give appropriate credit to the original author(s) and the source, provide a link to the Creative Commons licence, and indicate if changes were made. The images or other third party material in this article are included in the article's Creative Commons licence, unless indicated otherwise in a credit line to the material. If material is not included in the article's Creative Commons licence and your intended use is not permitted by statutory regulation or exceeds the permitted use, you will need to obtain permission directly from the copyright holder. To view a copy of this licence, visit http://creativecommons.org/licenses/by/4.0/.

\section{References}

1. Suares NC, Ford AC. Prevalence of, and risk factors for, chronic idiopathic constipation in the community: systematic review and meta-analysis. Am J Gastroenterol. 2011;106:1582-91 quiz 1581, 1592.

2. Damon H, Dumas P, Mion F. Impact of anal incontinence and chronic constipation on quality of life. Gastroenterol Clin Biol. 2004;28:16-20.

3. Dennison C, Prasad M, Lloyd A, Bhattacharyya SK, Dhawan R, Coyne K. The health-related quality of life and economic burden of constipation. Pharmacoeconomics. 2005;23:461-76.

4. Irvine EJ, Ferrazzi S, Pare P, Thompson WG, Rance L. Healthrelated quality of life in functional GI disorders: focus on constipation and resource utilization. Am J Gastroenterol. 2002;97:198693.

5. Sun SX, Dibonaventura M, Purayidathil FW, Wagner JS, Dabbous O, Mody R. Impact of chronic constipation on health-related quality of life, work productivity, and healthcare resource use: an analysis of the National Health and Wellness Survey. Dig Dis Sci. 2011;56: 2688-95.

6. McCallum IJ, Ong S, Mercer-Jones M. Chronic constipation in adults. BMJ. 2009;338:b831.

7. Ford AC, Moayyedi P, Lacy BE, et al. American College of Gastroenterology monograph on the management of irritable bowel 
syndrome and chronic idiopathic constipation. Am J Gastroenterol. 2014;109(Suppl 1):S2-26 quiz S27.

8. Johanson JF. Review of the treatment options for chronic constipation. MedGenMed. 2007;9:25.

9. Johanson JF, Kralstein J. Chronic constipation: a survey of the patient perspective. Aliment Pharmacol Ther. 2007;25:599-608.

10. Tamura A, Tomita T, Oshima T, Toyoshima F, Yamasaki T, Okugawa T, et al. Prevalence and self-recognition of chronic constipation: results of an internet survey. J Neurogastroenterol Motil. 2016;22:677-85.

11. Hirose T, Shinoda Y, Yoshida A, Kurimoto M, Mori K, Kawachi $\mathrm{Y}$, et al. Efficacy of daiokanzoto in chronic constipation refractory to first-line laxatives. Biomed Rep. 2016;5:497-500.

12. Jiang $\mathrm{C}, \mathrm{Xu} \mathrm{Q}$, Wen $\mathrm{X}$, Sun H. Current developments in pharmacological therapeutics for chronic constipation. Acta Pharm Sin B. 2015;5:300-9.

13. Japan Pharmaceutical and Medical Device Agency (PMDA). Linaclotide (Linzess ${ }^{\circledR}$ ). Prescribing information [Internet]. 2017 [updated August 2018]. Available from: http://www.pmda.go.jp/ PmdaSearch/iyakuDetail/ResultDataSetPDF/800126_ 2399017F1020 1 03\#page=3. Accessed October 17, 2018.

14. US Food and Drug Administration (FDA). Linaclotide (Linzess $\left.{ }^{\circledR}\right)$. Prescribing information [Internet]. 2012 [updated January 2017]. Reference ID: 4066835. Available from: https://www.accessdata. fda.gov/drugsatfda_docs/label/2017/202811s013lbl.pdf\#page=20. Accessed October 17, 2018.

15. Ford AC, Suares NC. Effect of laxatives and pharmacological therapies in chronic idiopathic constipation: systematic review and meta-analysis. Gut. 2011;60:209-18.

16. Lasa JS, Altamirano MJ, Bracho LF, Paz S, Zubiaurre I. Efficacy and safety of intestinal secretagogues for chronic constipation: a systematic review and meta-analysis. Arq Gastroenterol. 2018;55: $2-12$.

17. Ades AE, Sculpher M, Sutton A, Abrams K, Cooper N, Welton N, et al. Bayesian methods for evidence synthesis in cost-effectiveness analysis. Pharmacoeconomics. 2006;24:1-19.

18. Caldwell DM, Ades AE, Higgins JP. Simultaneous comparison of multiple treatments: combining direct and indirect evidence. BMJ. 2005;331:897-900.

19. Jansen JP, Crawford B, Bergman G, Stam W. Bayesian metaanalysis of multiple treatment comparisons: an introduction to mixed treatment comparisons. Value Health. 2008;11:956-64.

20. Lu G, Ades AE. Combination of direct and indirect evidence in mixed treatment comparisons. Stat Med. 2004;23:3105-24.

21. Nelson AD, Camilleri M, Chirapongsathorn S, Vijayvargiya P, Valentin N, Shin A, et al. Comparison of efficacy of pharmacological treatments for chronic idiopathic constipation: a systematic review and network meta-analysis. Gut. 2017;66:1611-22.

22. Tang W, Iwasaki K. Indirect comparison of linaclotide with other constipation treatments in patients with chronic constipation. PROSPERO 2018 CRD42018111737. 2018. Available from: http://www.crd.york.ac.uk/PROSPERO/display_record.php?ID= CRD42018111737 [database on the Internet]. Accessed: October 17, 2018.

23. Higgins JPT, Green S (editors). Cochrane Handbook for Systematic Reviews of Interventions Version 5.1.0 [updated March 2011]. The Cochrane Collaboration. 2011. Available from http://handbook. cochrane.org.

24. Cornell JE. The PRISMA extension for network meta-analysis: bringing clarity and guidance to the reporting of systematic reviews incorporating network meta-analyses. Ann Intern Med. 2015;162: 797-8.

25. Cochrane Controlled Register of Trials (CENTRAL) [database on the Internet]. John Wiley \& Sons Ltd. Available from: https://www. cochranelibrary.com/central/about-central. Accessed: October 17, 2018.
26. Higgins JPT, Sterne JAC, Savović J, et al. A revised tool for assessing risk of bias in randomized trials. Cochrane Database Syst Rev. 2016;10:29-31.

27. Hoaglin DC, Hawkins N, Jansen JP, Scott DA, Itzler R, Cappelleri $\mathrm{JC}$, et al. Conducting indirect-treatment-comparison and networkmeta-analysis studies: report of the ISPOR Task Force on Indirect Treatment Comparisons Good Research Practices: part 2. Value Health. 2011;14:429-37.

28. Jansen JP, Fleurence R, Devine B, Itzler R, Barrett A, Hawkins N, et al. Interpreting indirect treatment comparisons and network metaanalysis for health-care decision making: report of the ISPOR Task Force on Indirect Treatment Comparisons Good Research Practices: part 1. Value Health. 2011;14:417-28.

29. White IR, Barrett JK, Jackson D, Higgins JP. Consistency and inconsistency in network meta-analysis: model estimation using multivariate meta-regression. Res Synth Methods. 2012;3:111-25.

30. Fukudo S, Miwa H, Nakajima A, et al. A randomized controlled and long-term linaclotide study of irritable bowel syndrome with constipation patients in Japan. Neurogastroenterol Motil. 2018; e13444.

31. Fukudo S, Miwa H, Nakajima A, et al. High-dose linaclotide is effective and safe in patients with chronic constipation: a phase III randomized, double-blind, placebo-controlled study with a longterm open-label extension study in Japan. Neurogastroenterol Motil. 2018;e13487.

32. Fukudo S, Miwa H, Nakajima A, et al. Dose-finding study of linaclotide in Japanese patients with chronic constipation: a phase II randomized, double-blind, and placebo-controlled study. Neurogastroenterol Motil. 2018;e13442.

33. Fukudo S, Nakajima A, Fujiyama Y, Kosako M, Nakagawa A, Akiho $\mathrm{H}$, et al. Determining an optimal dose of linaclotide for use in Japanese patients with irritable bowel syndrome with constipation: a phase II randomized, double-blind, placebo-controlled study. Neurogastroenterol Motil. 2018;30:e13275.

34. Andresen V, Camilleri M, Busciglio IA, Grudell A, Burton D, McKinzie $\mathrm{S}$, et al. Effect of 5 days linaclotide on transit and bowel function in females with constipation-predominant irritable bowel syndrome. Gastroenterology. 2007;133:761-8.

35. Awad RA, Camacho S. A randomized, double-blind, placebocontrolled trial of polyethylene glycol effects on fasting and postprandial rectal sensitivity and symptoms in hypersensitive constipation-predominant irritable bowel syndrome. Colorectal Dis. 2010;12:1131-8.

36. Badiali D, Corazziari E, Habib FI, Tomei E, Bausano G, Magrini P, et al. Effect of wheat bran in treatment of chronic nonorganic constipation. A double-blind controlled trial. Dig Dis Sci. 1995;40: 349-56.

37. Barish CF, Drossman D, Johanson JF, Ueno R. Efficacy and safety of lubiprostone in patients with chronic constipation. Dig Dis Sci. 2010;55:1090-7.

38. Bouhnik Y, Neut C, Raskine L, Michel C, Riottot M, Andrieux C, et al. Prospective, randomized, parallel-group trial to evaluate the effects of lactulose and polyethylene glycol-4000 on colonic flora in chronic idiopathic constipation. Aliment Pharmacol Ther. 2004;19:889-99.

39. Chapman RW, Stanghellini V, Geraint M, Halphen M. Randomized clinical trial: macrogol/PEG 3350 plus electrolytes for treatment of patients with constipation associated with irritable bowel syndrome. Am J Gastroenterol. 2013;108:1508-15.

40. Chaussade S, Minic M. Comparison of efficacy and safety of two doses of two different polyethylene glycol-based laxatives in the treatment of constipation. Aliment Pharmacol Ther. 2003;17:16572.

41. Chey WD, Lembo AJ, Lavins BJ, Shiff SJ, Kurtz CB, Currie MG, et al. Linaclotide for irritable bowel syndrome with constipation: a 26-week, randomized, double-blind, placebo-controlled trial to 
evaluate efficacy and safety. Am J Gastroenterol. 2012;107:170212.

42. Chey WD, Paré P, Viegas A, Ligozio G, Shetzline MA. Tegaserod for female patients suffering from IBS with mixed bowel habits or constipation: a randomized controlled trial. Am J Gastroenterol. 2008;103:1217-25.

43. Chey WD, Webster L, Sostek M, Lappalainen J, Barker PN, Tack J. Naloxegol for opioid-induced constipation in patients with noncancer pain. N Engl J Med. 2014;370:2387-96.

44. Christie J, Shroff S, Shahnavaz N, Carter LA, Harrison MS, DietzLindo KA, et al. A randomized, double-blind, placebo-controlled trial to examine the effectiveness of lubiprostone on constipation symptoms and colon transit time in diabetic patients. Am J Gastroenterol. 2017;112:356-64.

45. Emmanuel AV, Roy AJ, Nicholls TJ, Kamm MA. Prucalopride, a systemic enterokinetic, for the treatment of constipation. Aliment Pharmacol Ther. 2002;16:1347-56.

46. Fukudo S, Hongo M, Kaneko H, Takano M, Ueno R. Lubiprostone increases spontaneous bowel movement frequency and quality of life in patients with chronic idiopathic constipation. Clin Gastroenterol Hepatol. 2015;13:294-301.e295.

47. Irving G, Pénzes J, Ramjattan B, Cousins M, Rauck R, Spierings $\mathrm{ELH}$, et al. A randomized, placebo-controlled phase 3 trial (Study SB-767905/013) of alvimopan for opioid-induced bowel dysfunction in patients with non-cancer pain. J Pain. 2011;12:175-84.

48. Jamal MM, Adams AB, Jansen JP, Webster LR. A randomized, placebo-controlled trial of lubiprostone for opioid-induced constipation in chronic noncancer pain. Am J Gastroenterol. 2015;110: 725-32.

49. Jansen JP, Lorch D, Langan J, Lasko B, Hermanns K, Kleoudis CS, et al. A randomized, placebo-controlled phase 3 trial (Study SB767905/012) of alvimopan for opioid-induced bowel dysfunction in patients with non-cancer pain. J Pain. 2011;12:185-93.

50. Johanson JF, Morton D, Geenen J, Ueno R. Multicenter, 4-week, double-blind, randomized, placebo-controlled trial of lubiprostone, a locally-acting type- 2 chloride channel activator, in patients with chronic constipation. Am J Gastroenterol. 2008;103:170-7.

51. Johanson JF, Wald A, Tougas G, Chey WD, Novick JS, Lembo AJ, et al. Effect of tegaserod in chronic constipation: a randomized, double-blind, controlled trial. Clin Gastroenterol Hepatol. 2004;2: 796-805.

52. Johnston JM, Kurtz CB, Drossman DA, Lembo AJ, Jeglinski BI, MacDougall JE, et al. Pilot study on the effect of linaclotide in patients with chronic constipation. Am J Gastroenterol. 2009;104: 125-32.

53. Johnston JM, Kurtz CB, Macdougall JE, et al. Linaclotide improves abdominal pain and bowel habits in a phase IIb study of patients with irritable bowel syndrome with constipation. Gastroenterology. 2010;139:1877-1886.e1872.

54. Kamm MA, Mueller-Lissner S, Wald A, Richter E, Swallow R, Gessner U. Oral bisacodyl is effective and well-tolerated in patients with chronic constipation. Clin Gastroenterol Hepatol. 2011;9:57783.

55. Kamm MA, Müller-Lissner S, Talley NJ, et al. Tegaserod for the treatment of chronic constipation: a randomized, double-blind, placebo-controlled multinational study. Am J Gastroeneterol. 2005; 100:362-72.

56. Kienzle-Horn S, Vix JM, Schuijt C, Peil H, Jordan CC, Kamm MA. Efficacy and safety of bisacodyl in the acute treatment of constipation: a double-blind, randomized, placebo-controlled study. Aliment Pharmacol Ther. 2006;23:1479-88.

57. Lacy BE, Schey R, Shiff SJ, Lavins BJ, Fox SM, Jia XD, et al. Linaclotide in chronic idiopathic constipation patients with moderate to severe abdominal bloating: a randomized, controlled trial. PLoS One. 2015;10:e134349.
58. Lembo AJ, Kurtz CB, Macdougall JE, et al. Efficacy of linaclotide for patients with chronic constipation. Gastroenterology. 2010;138: 886-895.e881.

59. Lembo AJ, Schneier HA, Shiff SJ, Kurtz CB, MacDougall JE, Jia $\mathrm{XD}$, et al. Two randomized trials of linaclotide for chronic constipation. N Engl J Med. 2011;365:527-36.

60. Heitland W, Mauersberger H. Study of the laxative effect of lactitol as opposed to lactulose in an open, randomized comparative study [article in German]. Schweiz Rundsch Med Prax. 1988;77:493-5.

61. Hongo M, Fukudo S, Kaneko H, Ueno T. Clinical evaluation of lubiprostone for chronic idiopathic constipation-double-blind, placebo-controlled, dose-finding study [article in Japanese]. J New Rem Clin. 2012;61:2471-87.

62. Sanders M, Jones S, Löwenstein O, Jansen JP, Miles H, Simpson K. New formulation of sustained release naloxone can reverse opioid induced constipation without compromising the desired opioid effects. Pain Med. 2015;16:1540-50

63. Shroff S, Shahnavaz N, Carter L, Harrison M, Dietz-Lindo K, Hanfelt J, et al. A randomized, double-blind controlled trial to examine the effectiveness of lubiprostone on constipation symptoms and colon transit time in diabetic patients. Am J Gastroenterol. 2015; 110:S739-40.

64. Sloots CE, Rykx A, Cools M, Kerstens R, De Pauw M. Efficacy and safety of prucalopride in patients with chronic noncancer pain suffering from opioid-induced constipation. Dig Dis Sci. 2010;55: 2912-21.

65. Sobhani I, Sansarricq M, Flourie B, et al. Laxative effect and tolerance of lactulose: one versus two intakes in patients with chronic constipation [article in French]. Gastroenterol Clin Biol. 1996;20: $362-6$.

66. Tomás-Ridocci M, Añón R, Mínguez M, Zaragoza A, Ballester J, Benages A. The efficacy of Plantago ovata as a regulator of intestinal transit. A double-blind study compared to placebo [article in Spanish]. Rev Esp Enferm Dig. 1992;82:17-22.

67. Webster L, Dhar S, Eldon M, Masuoka L, Lappalainen J, Sostek M. A phase 2, double-blind, randomized, placebo-controlled, doseescalation study to evaluate the efficacy, safety, and tolerability of naloxegol in patients with opioid-induced constipation. Pain. 2013;154:1542-50.

68. Xu Z, Dai J, Shi R, et al. A multicenter, randomized, single-blinded, parallel-controlled trial on lactitol in treatment of constipation [article in Chinese]. Chin J Gastroenterol. 2012;17:168-72.

69. Fenn GC, Wilkinson PD, Lee CE, Akbar FA. A general practice study of the efficacy of Regulan in functional constipation. Br J Clin Pract. 1986;40:192-7.

70. Lin S-R, Ke M-Y, Luo J-Y, Yuan YZ, Wang JY, diTommaso S, et al. A randomized, double-blind, placebo-controlled trial assessing the efficacy and safety of tegaserod in patients from China with chronic constipation. World J Gastroenterol. 2007;13: 732-9.

71. Mareya S, Drossman D, Joswick T, Dolecek G, Sun Y, Ueno R. Lubiprostone effectively relieves opioid-induced constipation in patients using non-diphenylheptane opioids for non-cancer pain: pooled analysis of three randomized controlled trials. Am J Gastroenterol. 2013;108:S567 [abstract].

72. Miner PB Jr, Koltun WD, Wiener GJ, de la Portilla M, Prieto B, Shailubhai K, et al. A randomized phase III clinical trial of plecanatide, a uroguanylin analog, in patients with chronic idiopathic constipation. Am J Gastroenterol. 2017;112:613-21.

73. Müller-Lissner S, Rykx A, Kerstens R, Vandeplassche L. A doubleblind, placebo-controlled study of prucalopride in elderly patients with chronic constipation. Neurogastroenterol Motil. 2010;22:9918 e255.

74. Novick J, Miner P, Krause R, Glebas K, Bliesath H, Ligozio G, et al. A randomized, double-blind, placebo-controlled trial of 
tegaserod in female patients suffering from irritable bowel syndrome with constipation. Aliment Pharm Ther. 2002;16:1877-88.

75. Paulson DM, Kennedy DT, Donovick RA, Carpenter RL, Cherubini M, Techner L, et al. Alvimopan: an oral, peripherally acting, mu-opioid receptor antagonist for the treatment of opioidinduced bowel dysfunction - a 21-day treatment-randomized clinical trial. J Pain. 2005;6:184-92.

76. Rauck R, Slatkin NE, Stambler N, Harper JR, Israel RJ. Randomized, double-blind trial of oral methylnaltrexone for the treatment of opioid-induced constipation in patients with chronic noncancer pain. Pain Pract. 2017;17:820-8.

77. ClinicalTrials.gov. Phase 2 randomized, double-blind, placebocontrolled, parallel group trial of linaclotide administered to patients with opioid-induced constipation receiving chronic opioid treatment for non-cancer pain. 2016. Available from: https:// clinicaltrials.gov/ct2/show/NCT02270983. Accessed November 12,2018

78. ClinicalTrials.gov. Dose-range-finding, phase 2 trial of oral linaclotide acetate administered to patients with chronic constipation. 2013. Available from: https://clinicaltrials.gov/ct2/ show/NCT00402337?term=NCT00402337\&rank=1. Accessed February 1, 2019.

79. ClinicalTrials.gov. Trial of linaclotide administered to patients with irritable bowel syndrome with constipation. 2013. Available from: https://clinicaltrials.gov/ct2/show/NCT00948818?term= NCT00948818\&rank=1. Accessed February 1, 2019.

80. ClinicalTrials.gov. A phase 3, international, multicenter, randomized, double-blind, placebo-controlled, parallel-group efficacy and safety trial of linaclotide administered orally for 12 weeks to patients with irritable bowel syndrome with constipation (IBS-C) (D5630C00001). 2016. Available from: https://clinicaltrials.gov/ ct $2 /$ show $/$ NCT0 1880424 ? term $=$ NCT0 $1880424 \&$ rank $=1$. Accessed February 1, 2019.

81. ClinicalTrials.gov. Efficacy and safety of lubiprostone in patients with irritable bowel syndrome with constipation. 2014. Available from: https://clinicaltrials.gov/ct2/show/NCT00380250?term= NCT00380250\&rank=1. Accessed February 1, 2019.
82. European Medicines Agency. Constella (linaclotide). Summary of product characteristics: [internet]. 2017. Available from: http:// www.Ema.Europa.Eu/docs/en_gb/document_library/epar__product_information/human/002490/wc500135622.pdf. Accessed April 27, 2020.

83. Portalatin M, Winstead N. Medical management of constipation. Clin Colon Rectal Surg. 2012;25:12-9.

84. Fukudo S, Hongo M, Kaneko H, Ueno R. Efficacy and safety of oral lubiprostone in constipated patients with or without irritable bowel syndrome: a randomized, placebo-controlled and dosefinding study. Neurogastroenterol Motil. 2011;23:544-e205.

85. Japan Pharmaceutical and Medical Device Agency (PMDA). Lubiprostone (Amitiza $\left.{ }^{\circledR}\right)$. Prescribing information [Internet]. 2012 [updated November 2018]. Available from: http://www. info.pmda.go.jp/go/pack/2359006M1025_2_05/. Accessed February 4, 2019.

86. US Food and Drug Administration (FDA). Plecanatide (Trulance ${ }^{\circledR}$ ). Prescribing information [Internet]. 2017 [updated January 2017]. Reference ID: 4044252. Available from: https:// www.accessdata.fda.gov/drugsatfda_docs/label/2017/208745lbl. pdf. Accessed October 17, 2018.

87. Center for Drug Evaluation and Research. Guidance for industry: irritable bowel syndrome - clinical evaluation of drugs for treatment. Food and Drug Administration, 2012. Available at: https:// www.fda.gov/downloads/Drugs/Guidances/UCM205269.pdf.

88. Mangel AW. What should be the primary end point in irritable bowel syndrome? Clin Invest. 2013;3:131-6.

89. Pannemans J, Vanuytsel T, Tack J. New developments in the treatment of opioid-induced gastrointestinal symptoms. United European Gastroenterol J. 2018;6:1126-35.

90. Rumman A, Gallinger ZR, Liu LWC. Opioid induced constipation in cancer patients: pathophysiology, diagnosis and treatment. Expert Rev Qual Life Cancer Care. 2016;1:25-35.

Publisher's Note Springer Nature remains neutral with regard to jurisdictional claims in published maps and institutional affiliations. 